\title{
A TRANSCENDENTAL VIEW OF THE SPACE OF ALGEBRAIC RIEMANN SURFACES
}

\author{
H. E. RAUCH ${ }^{1}$
}

The subject of my lecture is not a large-scale and general theory but rather a special problem in conformal mapping. By way of justification for bringing it to the attention of a broader audience I may cite, first of all, its deep nature and honorable origin - the problem originated in Riemann's memoir [46] of 1857 on algebraic functions, and after the passage of a century some definitive insight into it is only now emerging as a result of the collective efforts of a group of mathematicians. Secondly, it is intimately bound up with a variety of topics of more general interest: conformal mapping with particular reference to extremal problems, several complex variables, and partial differential equations, on the one hand; topology and algebraic geometry, on the other and, in particular aspects, even group representations and arithmetic.

1. Introduction and history. Briefly put, and in modern paraphrase, Riemann observed: (a) that for compact (algebraic) Riemann surfaces of genus $g=0$, i.e., simply connected, one has the analogue of the Riemann mapping theorem, that is, all such surfaces not only admit topological maps on one another but also conformal maps, in particular, on the Riemann sphere (all maps of surfaces in this paper are assumed to be homeomorphisms without further mention); (b) for $g>0$ it is no longer true that the existence of a map of one surface on another (equivalent to their having the same genus) implies the existence of a conformal map; and (c) as a partial compensation for (b) and substitute for (a) in case $g>0$, if one considers the conformal equivalence classes obtained by identifying surfaces which admit mutual conformal maps, these classes form not an isolated point as in (a) but in some sense or other a continuum of finite dimension, in fact, of dimension one for $g=1$ and $3 g-3$ for $g \geqq 2$. Riemann called a set of parameters for the continuum moduli (Riemann and his immediate successors often spoke cryptically of the moduli, as though some particular set of moduli were implicitly distinguished-this has not been borne out by the modern research to be described below).

An address delivered before the New York meeting of the Society on April 29, 1963, by invitation of the Committee to Select Hour Speakers for Eastern Sectional Meetings; received by the editors September 14, 1964.

1 Partially supported by NSF G-18929. 
The reader will notice immediately in (c) the vagueness of the statement and, indeed, the lapse into archaic language necessitated by this vagueness. Indeed, one must consider Riemann's observations, profound though they were, as being of heuristic character. The bulk of this lecture is devoted to an exposition of the results of recent successful attempts to get at least a precise qualitative picture of the structure of the set $M^{o}$ of conformal equivalence classes of surfaces of a given genus $g$ and thus clarify fully the nature of the phenomenon discovered by Riemann. A synthesis of these results can be stated roughly as follows: $M^{o}$ can be made into a topological space endowed with a certain complex analytic structure of complex dimension $3 g-3$ on which a set of moduli or more properly local moduli can be defined to be an admissible set of local coordinates near a point; and the structure of $M^{\circ}$, the modulus space, is "natural" in the sense that the various sets of parameters which had been proposed over the years as "moduli" can indeed be shown (nontrivially) to be so in the new precise sense.

The qualification "roughly" in the preceding paragraph conceals the essence of the matter, namely, that the structure of $M^{\circ}$ can only be defined and discussed after the construction of two auxiliary transcendentally defined covering spaces of $M^{\theta}$, the Teichmueller space $T^{v}$ and the Torelli space $J^{\sigma}$, whose descriptions will appear in $\S 2$. As it develops, both $T^{o}$ and $J^{o}$ are complex analytic manifolds, $T^{*}$ being a smooth covering of $\mathrm{J}^{a}$, while the latter is a branched covering of $M^{0}$; furthermore, the nature of the branching can be completely and explicitly described, in the course of which description it develops that, outside of certain exceptions for $g=1,2,3$, the points on $M^{o}$ under the branch locus of $\mathfrak{J}^{g}$ are nonuniformizable. In the vicinity of such points, then, it is meaningless to define moduli; hence, to be able to speak of moduli unexceptionally it is necessary to pass to one of the auxiliary covering manifolds $T^{g}$ or $3^{o}$. The remarkable conclusion thus emerges that the fundamental clarification of the moduli phenomenon is achieved not by Riemann's continuum $M^{a}$, even with its newly acquired structure, but by the new spaces $T^{o}$ and $\mathfrak{J}^{o}$. This is all the more noteworthy in that, following Riemann, the algebraic geometers had long dreamed of a purely algebraic construction by which $M^{\circ}$ would appear as an open subvariety of an irreducible projective algebraic variety of dimension $3 g-3$. After many false starts the construction of such a variety was achieved (cf. [9]) but subsequent to most of the transcendental results already outlined and at first dependent on them, with a purely algebraic construction finally materializing still more recently (cf. [34]). The point 
is, however, that whereas the variety $M^{o}$ is defined not only over any abstract field but even the ring of integers the construction of $T^{g}$ and $J^{\sigma}$ cannot be accomplished except by use of the complex field and transcendental methods, and even over the complex field the algebraic methods fail to give the insight into the structure of $M^{0}$ that the transcendental methods do. These remarks should serve to shed some light on the title of my lecture.

However, the establishment of qualitative structure theorems, no matter how satisfactory, for suitable spaces of Riemann surfaces does not at all exhaust the store of interesting and unanswered questions unearthed by Riemann's discovery of moduli. Rather, according to my way of thinking, those theorems form a conceptual substrate for the consideration of certain concrete analytical questions such as the relationships among various sets of moduli, applications of moduli to other function-theoretic questions and, in particular, what I like to call the problem of "numerical moduli"-systems of parameters (perhaps more than $3 g-3$ in number) globally defined on $M^{\circ}$ (or $T^{\theta}$ or $\left.\Im^{o}\right)$ and such that numerical equality between respective sets is necessary and sufficient for conformal equivalence of the corresponding surfaces.

In §2 I shall state the principal definitions and theorems whose totality constitutes the structural picture of the space of Riemann surfaces of which I have been speaking. In addition I shall include there some remarks on numerical moduli and also on some major unsolved problems. In \$3 I shall supply the reader with the materials, in the form of references to the literature or, where advisable, sketchy hints for gaining reasonable ideas of proofs for the results of $\$ 2$, supplying a little more detail for those arguments which either have not appeared or are not adequately described in the literature. Finally, in $\$ 4$ there is an account of some typical applications as envisaged in the preceding paragraph.

To conclude this section, some additional remarks by way of elaboration on the foregoing will serve to round out the historical picture and orient the reader toward the new research.

First, the situation for $g=1$ was already well understood in Riemann's time in the language of elliptic functions. Given two (origincontaining) lattices of parallelograms in the $z$ - and $z^{\prime}$-planes with primitive period pairs $\left(\omega_{1}, \omega_{2}\right)$ and $\left(\Omega_{1}, \Omega_{2}\right)$, respectively, with $\tau=\omega_{2} / \omega_{1}$, $\tau^{\prime}=\Omega_{2} / \Omega_{1}$, Im $\tau>0, \operatorname{Im} \tau^{\prime}>0$, the necessary and sufficient condition for the existence of a conformal map (origin preserving) of the $z$-plane on the $z^{\prime}$-plane taking $\left(\omega_{1}, \omega_{2}\right)$ onto $\left(\Omega_{1}, \Omega_{2}\right)$ is that $\tau=\tau^{\prime}$ in which case the map is necessarily given by $z^{\prime}=a z, a=\Omega_{1} / \omega_{1}=\Omega_{2} / \omega_{2}$. Thus the 
classes of "conformally equivalent period pairs" may be identified with the upper half of the $\tau$-plane and thus given the structure of a one-dimensional complex analytic manifold. As will be seen later, in terms of the compact Riemann surfaces of genus one obtained by identifying the plane under the action of the translation groups generated by the period pairs, this manifold is precisely the Teichmueller space $T^{1}$ and also the Torelli space $J^{1}$ (the two spaces coinciding in this, and only this, case, $g=1$ ). Furthermore, if one asks merely that the map $z^{\prime}=a z$ take $\left(\omega_{1}, \omega_{2}\right)$ onto some primitive period pair $\left(\Omega_{1}{ }^{\prime}, \Omega_{2}^{\prime}\right)$, not necessarily the preassigned one, then since $\Omega_{2}^{\prime}=a \Omega_{2}+b \Omega_{1}, \Omega_{1}^{\prime}=c \Omega_{2}+d \Omega_{1}, a, b, c, d$, integral and $a c-b d=1$, one has $\tau=\left(a \tau^{\prime}+b\right) /\left(c \tau^{\prime}+d\right)$. In the language of the associated Riemann surfaces this means (as it develops) that the set of conformal equivalence classes, i.e., $M^{1}$, is precisely the upper half-plane identified under the modular group. The modular invariant $J(\tau)$ maps a suitable standard fundamental domain representing $M^{1}$ one-one and conformally, except for the two vertices, on the sphere minus one point (corresponding to the cusp). That is the whole story for $g=1$, and in retrospect one sees that it contains several clues to the general situation, $g \geqq 2$, but historically that was not evident.

Next, whence came the $3 g-3$, for $g \geqq 2$ ? Riemann gave two counts, the second of which is incorporated as part of Prescription II of \$2 and finds its justification there. The first counts the "essential" number of branch points in a branched representation with sufficiently many sheets $n(>2 g-2)$ and arrives at $3 g-3=2(n+g-1)-n$ $-(n-g+1)$, where the first number is the total number of branch points, $n$ is also the number of poles of a representing function to be chosen arbitrarily, and the last number is the Riemann-Roch count of the number of linearly independent functions with only the prescribed poles giving rise to conformally equivalent surfaces. The first justification for this or any other method of using branch points as local moduli is found in [43], but its historical significance lies not only in the discovery of $3 g-3$ but in the impetus it gave to the algebraic geometers to attempt to construct a modulus variety of suitably normalized concrete representatives of surfaces (normal curves) and thus consciously formulate the notion of space of surfaces.

Another, very graphic and very useful, even in the current view of things, method of obtaining the count $3 g-3$ is uniformization by means of Fuchsian groups. The groups in question are generated by $2 g$ transformations $A_{1}, B_{1}, \ldots, A_{\boldsymbol{\theta}}, B_{\boldsymbol{\theta}}$, satisfying one relation $A_{1} B_{1} A_{1}^{-1} B_{1}^{-1} \cdots A_{0} B_{0} A_{g}^{-1} B_{0}^{-1}=1$. Each generator (being a fractional linear transformation of the unit disk on itself) has three un- 
normalized real parameters whence, subtracting three parameters fixed by the relation and three more for a normalization of the entire group, one obtains $3 \cdot 2 g-3-3=6 g-6$, the requisite number of real parameters. This remark led Klein and Poincaré to speculate on the possibility of a continuity proof for the uniformization of algebraic Riemann surfaces, the idea being that the "space" of such surfaces has, according to Riemann, the same dimension, $3 g-3$, as the set of (hopefully) uniformizing groups. Serious efforts at justifying this argument were made by Brouwer, Fricke, and Koebe (see [24] for an interesting account of these attempts circa 1911). Brouwer emphasized the topological difficulties, and his efforts to overcome them led to some of his significant contributions to topology. The most ambitious attack, embodied in the two-volume work [17], was due to Fricke and led to the construction of an auxiliary space resembling the later Teichmueller space. The length and complexity of Fricke's arguments have contributed to their being eclipsed by the much simpler and more lucid construction due to Teichmueller. For a modern account of a satisfactory approach to continuity proofs of uniformization theorems using recent research I refer the reader to [12]. A more modern version of Fricke's arguments exists, I am told, in an unpublished manuscript by Fenchel and Nielsen (cf. [19] which also contains applications of this method). The reader is also referred to [31] and references there.

The next stage was the work of Teichmueller who, in [53] and [54], combined in a surprising way two apparently unrelated ideas connected with moduli to make a decisive break-through. His first observation (already known to Riemann) was that $3 g-3$ (as one easily computes from the Riemann-Roch theorem) is the number of everywhere analytic quadratic differentials on a surface of genus $g$ which are linearly independent over the complex field. The other idea, based on ideas of Grötzsch, is to consider, given two surfaces of genus $g, S$ and $S^{\prime}$, the possibility of their being conformally equivalent by considering in each homotopy class of orientation-preserving homeomorphisms of $S$ on $S^{\prime}$ the infimum of the dilations of quasiconformal mappings in the class. The linking of these ideas leads to the following, collectively known as Teichmueller's theorem: There exists in each homotopy class a unique extremal quasi-conformal homeomorphism with constant minimal dilation $D \geqq 1$. If $D=1, S$ and $S^{\prime}$ are conformally equivalent by a map in the class. If $D>1$ there is associated (essentially) uniquely a pair of analytic quadratic differentials, one on $S$, the other on $S^{\prime}$, by means of which in suitable local coordinates the extremal map can be simply and explicitly represented 
as a locally affine map. For an account of Teichmueller's theorem see, besides [1], [11] and [53]. The intuitive part of this theorem, the map in the homotopy class, led Teichmueller to the definition of the set $T^{\circ}$ (vide $\$ 2$ ) covering $M^{\circ}$. The subtle part, the connection with quadratic differentials, led him to the definition of a locally euclidean structure of dimension $6 g-6$ (the real dimension of the space of quadratic differentials) which can be loosely described by saying that any element of $T^{\circ}$ can be obtained from a fixed one by "deforming along a quadratic differential" on the latter. In fact Teichmueller proved much more: using $\log D$ as a metric, he gave $T^{\circ}$ the structure of metric space and then, using the uniqueness assertion of his theorem, he proved that this Teichmueller space $T^{o}$ is homeomorphic to the euclidean $R^{6 \sigma-6}$. Oddly enough, this beautiful result, the globally euclidean nature of $T^{\circ}$, has, up to the present writing, not played any decisive role in subsequent research in the directions with which I am concerned in this exposition. Only the locally euclidean, or manifold, character of $T^{\circ}$ is essential. Here a technical point of great importance arises. Bers in [10] has shown that a suitable use of Teichmueller's ideas, in particular, a flexible use of quasi-conformal mapping, leads to the same local structure on $T^{\sigma}$ without the use of Teichmueller's theorem. This is a considerable technical and conceptual simplification in that the difficult proofs of existence and uniqueness of an extremal map are avoided. The uniqueness proof dispensed with uses a completely different order of ideas which are not immediately relevant to the applications, while the existence proof is replaced by a general treatment of the solution theory of a certain linear partial differential equation. The latter simplification is somewhat illusory in that the solution theory applied full force is sufficient to prove the existence part of Teichmueller's theorem. Another difficulty is that while one can dispense with Teichmueller's theorem in discussing the structure of $T^{o}$, I find that, at least for the present, I need one consequence of the theorem in treating the structure of $M^{o}$. I have organized the exposition accordingly, making no use of the theorem until needed.

Now Teichmueller observed that his set $T^{0}$ is a covering of Riemann's set $M^{0}$ in the precise sense that $M^{0}$ is the quotient of $T^{0}$ by the action of a geometrically defined group $\Gamma^{0}$, the mapping-class group, of 1-1 self-maps of $T^{0}$ and that, in his topology on $T^{o}, \Gamma^{o}$ acts as a properly discontinuous group of homeomorphisms (in fact, isometries in the metric). The result is that $M^{0}$ is endowed with the structure of a topological space such that $M^{0}$ minus the points that are covered by fixed points of $\Gamma^{0}$ is a $(6 g-6)$-dimensional manifold 
(the first complete proofs of these statements are in [25]).

Teichmueller's work left two unanswered questions about the space $M^{o}$ : (1) What is the topological character (in particular, manifold or nonmanifold) at the above exceptional points? (2) Does it have a complex structure, and, if so, does it have one with a natural relationship to his structure? The first part of (2) is critical in the realization of the program initiated by Riemann's work while the second is critical for the role of Teichmueller's proposals in that realization. Happily, despite Teichmueller's conjecture to the contrary, the answer to the latter part and hence all of (2) is affirmative, and a complete and explicit response to (1), intimately related to the complex structure of (2), has been given.

The answers to these questions have been the major concern, leaving aside the matter of applications, of the current phase of research into the topic at hand and, in particular, of my own contributions thereto. This phase began in 1955 with the notes [41] and [42] in which I gave prescriptions for finding sets (each containing $3 g-3$ elements) of periods of normal abelian integrals of first kind serving as local moduli near a given nonhyperelliptic surface (in fact, local coordinates on $T^{o}$ near suitable points covering the surface class) and analogous sets of $2 g-1$ elements each serving as local coordinates on the hyperelliptic locus of $T^{\circ}$ near a point thereof. The motivating idea behind introducing the periods was an attempt to refine an important theorem of Torelli (circa 1912: for some recent treatments see [8], [32] and [55]) to the effect that two surfaces with the same period matrices are conformally equivalent (more precision in \$2). Torelli's theorem furnishes a beautiful set of global moduli and gives rise (see \$2) to a second naturally defined covering of $M^{\circ}$, the Torelli space, which provides a natural setting for them, the only trouble being that the essential periods are superfluous in number, $g(g+1) / 2$, in contrast to $3 g-3$. My work picks out the correct number locally but not globally. Ahlfors [2], using my prescriptions and adding a vital ingredient of his own for the hyperelliptic surfaces, compounded the first proof of the existence of a natural structure of complex analytic manifold on Teichmueller's space $T^{\circ}$. Subsequent proofs of the existence of an equivalent structure dispensing with periods and the consequent special handling of the hyperelliptic surfaces were given, the equivalence resulting from my work and Ahlfors'. Of these the one due to Bers [10] is the most simple and useful and in the hands of Bers and Ahlfors it has become a powerful tool for studying $T^{\circ}$ and more general Teichmueller-type spaces for Riemann surfaces which are uniformized by larger classes of groups than the 
special Fuchsian groups of the compact surfaces. For an account of this latter work I must refer the reader to current publications of these authors. Here, I intend to retain a discussion of the original version of the complex structure for the simple reason that the periods are the sole link with those applications which I mentioned earlier as forming a major justification for the theory.

The relevance of the new-found complex structure on $T^{\theta}$ to the structure of $M^{0}$ stems from the convenient fact that $\Gamma^{o}$ now appears as a group of complex analytic homeomorphisms. Hence, by a nowstandard process [15], $M^{0}$ as the quotient of $T^{o}$ by $\Gamma^{o}$ now is endowed with the structure of normal analytic space-the long-sought goal in precise form. Furthermore, there is additional precision. The nature of the structure of $M^{0}$ at the points covered by the fixed points of $\Gamma^{o}$ (all other points being manifold points) yields to analysis by virtue of two facts. The first, due to Teichmueller, is that at a point $t$ in $T^{o}$ the isotropy subgroup of $\Gamma^{0}$ is canonically anti-isomorphic to the group $H(S)$ of conformal automorphisms of a surface $S$ in the class represented by the point of $M^{\sigma}$ under $t$. The second, a property of the new complex structure on $T^{\sigma}$, is that the analysis of the structure of $M^{0}$ at the point in question is equivalent to the analysis of the representation of $H(S)$ on the (quadratic) differentials of $S$. Here one is brought into contact with a beautiful and profound meeting of analysis and arithmetic originated by Hurwitz [22] and carried on by Hecke [21]. In this connection I would like to mention the work of J. Lewittes [28], [29] which arose in this context and in turn contributed to my own understanding of it. The first crude level of analysis already brings out that the points in question (except for some cases in genera 2 and 3) are all nonmanifold points [27], [45] justifying the earlier assertion about the unsuitability of the modulus space $M^{\sigma}$ as a testing ground for sets of local moduli.

To conclude this informal outline let me first cite [7], [35], [39], [40], [52], and [57] and references there for those who may want to read up on those parts of Riemann surface theory relevant here, and finally let me direct the reader's attention to the recently developing inquiry into moduli in several complex variables where the situation is more complex and less completely investigated. Here, a few citations of authors and approaches will have to suffice: using harmonic integrals, Kodaira and Spencer [23], Kuranishi [26]; using Weil's approach to abelian varieties, Shimura [51]; using coherent analytic sheaves, Grauert [18]; and using his general program of schemes, Grothendieck [20]. 
2. Definitions and theorems. All references to literature and indications of proof for the results of $\$ 2$ are deferred to $\$ 3$.

A. Certain sets, maps and groups. Henceforth, the letter $S$ with any combination of subscripts or superscripts will denote a compact Riemann surface of genus $g(\geqq 0)$, and all maps of compact Riemann surfaces will be orientation-preserving homeomorphisms.

I consider triples $\left(S, S^{\prime}, \alpha\right)$, where $\alpha: S \rightarrow S^{\prime}$. For maps of $S$, the first member of a triple, the symbols $\sim, \approx$, and 1 will denote, respectively, "homotopic," "inducing the same automorphism of $H_{1}(S)$ (the first homology group)," and the identity. Consider, in particular, the three notions of equivalence of triples:

Definition 1. (a) $\left(S_{1}, S_{1}^{\prime}, \alpha_{1}\right) \sim\left(S_{2}, S_{2}^{\prime}, \alpha_{2}\right)$, read "Teichmuellerequivalent," $\Leftrightarrow S_{1}=S_{2}$ and there exists an $f: S_{1}^{\prime} \rightarrow S_{2}^{\prime}, f$ conformal, such that $f \sim \alpha_{2} \alpha_{1}^{-1}$.

(b) $\left(S_{1}, S_{1}^{\prime}, \alpha_{1}\right) \approx\left(S_{2}, S_{2}^{\prime}, \alpha_{2}\right)$, read "Torelli-equivalent," $\Leftrightarrow$ same as (a) except $\sim$ replaced by $\approx$.

(c) $\left(S_{1}, S_{1}^{\prime}, \alpha_{1}\right) \equiv\left(S_{2}, S_{2}^{\prime}, \alpha_{2}\right)$, read "conformally equivalent," $\Leftrightarrow$ same as (a) with the deletion of the phrase after "conformal."

$\left(a^{\prime}\right)$ A class of Teichmueller-equivalent triples containing $\left(S, S^{\prime}, \alpha\right)$ with common first member $S$ is a Teichmueller surface $(S)$ denoted by $\left\langle S, S^{\prime}, \alpha\right\rangle$.

$\left(b^{\prime}\right)$ Substitution of "Torelli-equivalent" for "Teichmueller-equivalent" in $\left(\mathrm{a}^{\prime}\right)$ defines a Torelli surface $(S)$ denoted by $\left\{S, S^{\prime}, \alpha\right\}$.

$\left(c^{\prime}\right)$ Similar substitution of "conformally-equivalent" in $\left(a^{\prime}\right)$ defines a conformal equivalence class denoted by $\left[S^{\prime}\right]$ (the dependence on $S$ being now irrelevant).

$\left(\mathrm{a}^{\prime \prime}\right) T^{\circ}(S)$ is the totality of Teichmueller surfaces $(S) .\langle S, S, 1\rangle$ is the origin of $T^{\circ}(S)$.

$\left(\mathrm{b}^{\prime \prime}\right) \mathfrak{J}^{\circ}(S)$ is the totality of Torelli surfaces $(S) .\{S, S, 1\}$ is the origin of $\mathfrak{J}^{0}(S)$.

(c') $M^{0}$ is the totality of conformal equivalence classes.

It will be noticed that in the present exposition the sets $T^{o}(S)$ and $J^{o}(S)$ are not unique, being indexed by $S$. This turns out to be very convenient when coupled with the fact, to be developed subsequently, that there is a natural set of one-one maps of $T^{\circ}\left(S_{1}\right)\left(J^{o}\left(S_{1}\right)\right)$ on $T^{\circ}\left(S_{2}\right)\left(\mathcal{J}^{\circ}\left(S_{2}\right)\right)$ for arbitrary $S_{1}, S_{2}$, which maps preserve all structures to be imposed on those sets. On the other hand, it is not possible to single out one of these maps canonically. It will be possible to speak of sets $T^{\circ}$ and $J^{0}$ only by the same abuse of language that permits one to speak of the fundamental group of a space.

Definition 2. Given $S, S^{\prime}$ and a map $\alpha: S \rightarrow S^{\prime}$, the maps $\alpha^{*}: T^{\circ}\left(S^{\prime}\right)$ $\rightarrow T^{\circ}(S)$ and $\alpha^{*}: J^{o}\left(S^{\prime}\right) \rightarrow J^{\circ}(S)$ defined, respectively, by $\alpha^{*}\left\langle S^{\prime}, S^{\prime \prime}, \beta\right\rangle$ 
$=\left\langle S, S^{\prime \prime}, \beta \alpha\right\rangle$ and $\alpha^{\sharp}\left\{S^{\prime}, S^{\prime \prime}, \beta\right\}=\left\{S, S^{\prime \prime}, \beta \alpha\right\}$ are called admissible. For $S=S^{\prime}$ the set of all admissible self-maps of $T^{\circ}(S)$, respectively $\mathfrak{J}^{o}(S)$, is denoted by $\Gamma^{o}(S)$, respectively $\mathfrak{M}^{o}(S) . \Delta^{o}(S) \subset \Gamma^{o}(S)$ denotes the subset consisting of those $\alpha^{*} \in \Gamma^{o}(S)$ for which the self-maps $\alpha$ of $S$ satisfy $\alpha \approx 1$.

Proposition 1. $\alpha \sim \beta \Rightarrow \alpha^{*}=\beta^{*} . \alpha \approx \beta \Rightarrow \alpha^{*}=\beta^{*}$. All admissible maps are one-one onto, $\Gamma^{\circ}(S)$ and $\mathfrak{M}^{\circ}(S)$ can be given natural structures as groups of one-one self-maps of $T^{o}(S)$ and $\jmath^{g}(S)$, respectively. $\Delta^{o}(S)$ is a fixed-point-free normal subgroup. With these structures one has $\jmath^{\circ}(S)$ $=T^{o}(S) / \Delta^{o}(S)$ and $M^{o}=T^{o}(S) / \Gamma^{o}(S)=J^{o}(S) / \mathfrak{M}^{o}(S)$, where the quotient maps $T^{o}(S) \rightarrow J^{\circ}(S) \rightarrow M^{\circ}$ thus defined coincide with the natural projections obviously defined by Definition 1 , and one has $\Gamma^{o}(S) / \Delta^{o}(S)$ $=\mathfrak{M}^{0}(S)$.

B. Metric, topological, and analytic structures for $T^{\circ}(S)$ and $\jmath^{\circ}(S)$.

Definition 3. A Beltrami differential $\mu$, respectively, a quadratic differential $\phi$, respectively, a density $\lambda$ on $S$, is a tensor such that $\mu d \bar{z} / d z$ is invariant and its representatives are essentially bounded measurable functions, respectively, such that $\phi d z^{2}$ is invariant and its representatives are holomorphic (and finite), respectively, such that $\lambda d z d \bar{z}$ is invariant and its representatives are continuous positive functions. Observing that $|\mu|$ is invariant, one defines $\|\mu\|=\operatorname{ess} \sup |\mu|$, the sup taken over $S$. A proper Beltrami differential is one for which $\|\mu\|<1$.

Definition 4. A map $\alpha: S \rightarrow S^{\prime}$ is quasiconformal if, when represented as $w=w(z, \bar{z})$ in local coordinates, it satisfies the Beltrami equation $w_{z}=\mu w_{z}$ for some proper Beltrami differential $\mu$. The derivatives are to be taken in the conventional sense if $\alpha$ is $C^{1}$, otherwise in the generalized sense (see $\$ 3$ ). Define $k(\alpha) \equiv\|\mu\|$ and $K(\alpha)$ $=(1+k(\alpha)) /(1-k(\alpha))$.

Lemma 1. Given $\alpha: S \rightarrow S^{\prime}$, there exists $\beta: S \rightarrow S^{\prime}$ which is homotopic to $\alpha$ and quasiconformal (Definition 4).

Theorem 1. By defining, for each $S, D_{S}\left(\left\langle S, S^{\prime}, \alpha\right\rangle,\left\langle S, S^{\prime \prime}, \beta\right\rangle\right)$ $=\inf \log K(\gamma)$, where inf is over all quasiconformal maps $\gamma: S^{\prime} \rightarrow S^{\prime \prime}$, where $\gamma \sim \beta \alpha^{-1}$, one obtains for each $S$ a metric, the Teichmueller metric $(S)$ on $T^{\circ}(S)$. The induced topology is called the Teichmueller topology $(S)$ and $T^{\circ}(S)$ with this topology is the Teichmueller space $(S) . T^{\circ}(S)$ is connected in this topology. $\Gamma^{\circ}(S)$ (Definition 2 and Proposition 1) is a properly discontinuous group of isometries (in particular, homeomorphisms) in the Teichmueller metric (S) and topology (S). In particular (Proposition 1) one can put the quotient topology on $\Im^{\circ}(S)$ to define the 
Torelli space $(S)$ on which $\mathfrak{M}^{\circ}(S)$ is a properly discontinuous group of homeomorphisms. All admissible maps (Definition 2) of $T^{\circ}(S)$ on $T^{\circ}\left(S^{\prime}\right)$ are isometries. All admissible maps of $\mathrm{J}^{\circ}(S)$ on $\mathrm{J}^{\circ}\left(S^{\prime}\right)$ are homeomorphisms.

The following proposition plays no role in the development of the theory as outlined here but is included because of its intrinsic interest.

Proposition 2. $T^{\circ}(S)$, the Teichmueller space $(S)$, is homeomorphic to $R^{60-6}$, i.e., $(6 g-6)$-dimensional euclidean space, for $g \geqq 2$ (respectively, to $R^{2}$ for $g=1$, while $T^{0}(S)$ consists of one point).

The parenthetical statements are, in essence, well known and will be dealt with again later on.

LEMma 2. Given a proper Beltrami differential $\mu$ on $S$, in a sufficiently small neighborhood of any pre-assigned point on $S$ the associated Beltrami equation (Definition 4) has a solution which maps the neighborhood homeomorphically onto a plane domain. Any two such solutions in their common domain are complex analytic functions of one another with nonvanishing derivative.

Definition 5. Given $S$ and a proper Beltrami differential $\mu, S^{\mu}$ is the Riemann surface whose topological base space is that of $S$ and whose local parameters are the homeomorphic solutions of the Beltrami equation formed with $\mu$ (cf. Lemma 2). In particular, the identity map 1: $S \rightarrow S^{\mu}$ is quasiconformal.

Proposition 3. Let $B(S)$ be the linear space over $\boldsymbol{C}$ of all Beltrami differentials on $S$ with the norm of Definition 3. Let $A(S)$ be the linear space over $C$ of all quadratic differentials on $S . A(S)$ has dimension $3 g-3$ over $C$ for $g \geqq 2,1$ for $g=1$ and 0 for $g=0$. Define the bilinear functional $(\phi, \mu) \equiv(i / 2) \iint_{S} \phi \mu d z \wedge d \bar{z}$, where $\phi \in A(S), \mu \in B(S)$, and $\wedge$ denotes exterior product; and let $N(S) \subset B(S)$ be defined by $(\phi, \mu)=0$ for all $\phi \in A(S)$. There exists a density $\lambda$ on $S$. If $\phi \in A(S), \Phi / \lambda \in B(S)$ and the map defined by $\phi \rightarrow \Phi / \lambda$ is an anti-linear isomorphism of $A(S)$ on $B(S) / N(S)$; in particular, a basis of $B(S) / N(S)$ consists of $\mu_{i} \in B(S)$, $i=1, \cdots, 3 g-3$, for $g \geqq 2$, such that $\left(\phi, c_{1} \mu_{1}+\cdots+c_{3 g-3} \mu_{3 g-3}\right)=0$ for all $\phi \in A(S), c_{i} \in C, i=1, \cdots, 3 g-3$, implies $c_{1}=\cdots=c_{3 g-3}=0$, and there exists a basis. For $g=1$ replace $3 g-3$ by 1 . For $g=0$ the statement is vacuous.

Theorem 2. $T^{\circ}(S)$, the Teichmueller space $(S)$ (Theorem 1), when endowed with the local coordinates of Prescription I below, becomes a 
complex analytic manifold whose complex dimension is $3 g-3$ for $g \geqq 2$, 1 for $g=1$, and 0 for $g=0$. In this structure, $\Gamma^{g}(S)$, in addition to those properties listed in Theorem 1, has the property that each of its elements is complex analytic (with Jacobian never zero). By virtue of the fixedpoint-free character of $\Delta^{o}(S)$ (Proposition 1), for sufficiently small neighborhoods, $\mathfrak{J}^{\circ}(S)$ can be given the same coordinates by means of the natural projection $T^{o}(S) \rightarrow \Im^{o}(S)$ in such a way that it, too, becomes a complex analytic manifold and the projection becomes complex analytic. In this structure, $\mathfrak{R}^{\circ}(S)$ will then also be realized by complex analytic maps.

Prescription I. Let $\mu_{1}, \cdots, \mu_{30-3}$ be a basis of $B\left(S^{\prime}\right) / N\left(S^{\prime}\right)$ (Proposition 3), and let $c=\left(c_{1}, \cdots, c_{3 g-3}\right)$ be a vector in $C^{3 g-3}$, complex vector space of complex dimension $3 g-3$, for $g \geqq 2$; for $g=1$, replace $3 g-3$ by 1 . For $\left|c_{1}\right|^{2}+\cdots+\left|c_{3 g-3}\right|^{2}$ sufficiently small, $\mu \equiv c_{1} \mu_{3}+\cdots+c_{30-3} \mu_{30-3}$ is a proper Beltrami differential and the map $c \rightarrow\left\langle S, S^{\prime \mu}, \alpha\right\rangle$ (Lemma 2) is a homeomorphism of a neighborhood of the origin in $C^{3 a-3}$ on a neighborhood of $\left\langle S, S^{\prime}, \alpha\right\rangle \in T^{o}(S)$. The vectors in the first neighborhood are by prescription a set of coordinates for the second.

The following closely related proposition is, again, quoted because of its intrinsic interest although it plays no role in the developments at hand, and, again, the case $g=1$ is known.

Proposition 4. $T^{\circ}(S)$ with the structure of Theorem 2 is complex analytically homeomorphic to a bounded domain of $\mathrm{C}^{30-3}$ for $g \geqq 2$; of $C^{1}$, for $g=1$. This homeomorphism is not complex analytically equivalent to the homeomorphism of Proposition 2; indeed the latter is not complex analytic on $T^{g}(S)$ even if one imposes the structure of $C^{30-3}$ on $R^{60-6}$.

There is an alternative mode of introducing the same, i.e., complex analytically equivalent structures on the Teichmueller space $(S)$ and the Torelli space $(S)$, which mode as observed in $\$ 1$ is both more useful for certain applications and historically antecedent to that of Prescription I. As a preliminary to formulating the alternative in Prescription II below I shall recall certain standard facts about Riemann surfaces.

I shall write $(\gamma, \delta)=\left(\gamma_{1}, \cdots, \gamma_{\theta} ; \delta_{1}, \cdots, \delta_{\sigma}\right)$ to denote a canonical (one-dimensional) homology basis on $S$ where the one-cycles satisfy $K I\left(\gamma_{i}, \gamma_{j}\right)=0, K I\left(\delta_{i}, \delta_{j}\right)=\delta_{i j}, i, j=1, \cdots, g, K I$ denoting the (skewsymmetric, bilinear, integral-valued) intersection number. By abuse of language I shall write $(\gamma, \delta)=\left(\gamma^{\prime}, \delta^{\prime}\right)$ when the two bases are element-wise homologous. Given $(\gamma, \delta)$ on $S$, the normal abelian differentials of first kind $d \zeta_{1}, \cdots, d \zeta_{\theta}$ with respect to $(\gamma, \delta)$ on $S$ (I sup- 
press the dependence of $d \zeta_{i}$ on $S$ and $\left.(\gamma, \delta)\right)$ are uniquely defined by

(1a) $\int_{\gamma_{i}} d \zeta_{j}=\delta_{i j}, i, j=1, \cdots, g$, whence

(1b) $\pi_{i j}[S,(\gamma, \delta)] \equiv \int_{\delta_{j}} d \zeta_{i}, i, j=1, \cdots g$,

are uniquely determined. The $g \times g$ matrix $\pi[S,(\gamma, \delta)]=\pi_{i j}[S,(\gamma, \delta)]$ is symmetric with positive-definite imaginary part and thus is a point in $\mathfrak{S}_{\rho}$, the Siegel upper half-plane of genus $g$, the totality of such matrices. As a subset of $C^{o(o+1) / 2}, \mathfrak{S}_{\rho}$ inherits a natural structure of complex analytic manifold.

$S$ is hyperelliptic, for $g \geqq 2$, if and only if $S$ admits a two-sheeted representation or, equivalently, there exists a conformal self-map, $J$, of $S$, which is involutive, $J^{2}=1$, and has precisely $2 g+2$ fixed points. $J$, the "sheet interchange," is uniquely characterized by these properties.

The following lemma is a special case of a more general one which will appear later on, but its distinct formulation here is useful.

LEMma 3. Given a canonical homology basis $(\gamma, \delta)$ on $S$ and $\alpha: S \rightarrow S^{\prime}$, let $\alpha(\gamma, \delta)$ denote the canonical basis on $S^{\prime}$ which is the image of $(\gamma, \delta)$ under $\alpha$. Every $S$ admits an involutive self-map $\alpha$ such that $\alpha(\gamma, \delta)$ $=(-\gamma,-\delta)$. However, for $g \geqq 2$, when $S$ is hyperelliptic, and only then, does there exist such an $\alpha$ which is conformal, and then $\alpha=J$, the sheet interchange.

Inspection of equations (1a), (1b) yields immediately the trivial:

LEMMA 4. The normal differentials of the first kind on $S$ with respect to $(-\gamma,-\delta)$ are $-d \zeta_{i}, i=1, \cdots, g$ and $\pi[S,(-\gamma,-\delta)]=\pi[S,(\gamma, \delta)]$.

Definition 5. Let $U^{o}(S)$, respectively, $\mathcal{u}^{g}(S), g \geqq 2$, denote the hyperelliptic sublocus of $T^{o}(S)$, respectively, $\jmath^{o}(S)$, i.e., the totality of $\left\langle S, S^{\prime}, \alpha\right\rangle \in T^{o}(S)$, respectively, $\left\{S, S^{\prime}, \alpha\right\} \in J^{g}(S)$, such that $S^{\prime}$ is hyperelliptic. $T^{2}(S)=U^{2}(S) ; \mathfrak{J}^{2}(S)=\mathcal{u}^{2}(S)$.

The following proposition has played a fundamental role historically in motivating the introduction of Torelli space and the study of periods of abelian integrals as moduli.

Proposition 5. Given a canonical homology basis $(\gamma, \delta)$ on $S, g \geqq 2$, define a map ${ }^{\sharp} \pi: \mathfrak{J}^{\circ}(S) \rightarrow \mathfrak{S}_{0}$ (I suppress the dependence of ${ }^{\sharp} \pi$ on $(\gamma, \delta)$ since it will not cause any difficulty) by ${ }^{*} \pi\left\{S, S^{\prime}, \alpha\right\}=\pi\left[S^{\prime}, \alpha(\gamma, \delta)\right]$ (cf. equation (1b)). ${ }^{\#} \pi$ is 2-1 on $\jmath^{g}(S)-\mathcal{u}^{g}(S)$ and 1-1 on $\mathcal{u}^{g}(S)$. Indeed, ${ }^{\sharp} \pi\left\{S, S^{\prime}, \beta\right\}={ }^{\#} \pi\left\{S, S^{\prime \prime}, \beta^{\prime}\right\} \Leftrightarrow\left\{S, S^{\prime}, \beta\right\}=\left\{S, S^{\prime \prime}, \beta^{\prime}\right\}$ or $\left\{S, S^{\prime \prime}, \alpha \beta^{\prime}\right\}$, where $\alpha$ is the map of Lemma 3 and (Lemma 3 and Definition 1) $\left\{S, S^{\prime \prime}, \alpha \beta^{\prime}\right\}=\left\{S, S^{\prime \prime}, \beta^{\prime}\right\} \Leftrightarrow S^{\prime \prime}$ (and hence $S^{\prime}$ ) is hyperelliptic. 
Lemma 5. If $S, g>2$, is not hyperelliptic and $(\gamma, \delta)$ is a canonical homology basis on it, there exists a set $I(S)$ of $3 g-3$ ordered pairs $(i, j)$, $i, j$, integral and $1 \leqq i, j \leqq g$, such that $A(S)$ is generated by $d \zeta_{i} d \zeta_{j}$, $(i, j) \in I(S)$, where $d \zeta_{i}$ is the ith normal differential of first kind with respect to $(\gamma, \delta)$. For $g=1, A(S)$ is generated by the square of the unique normal differential, and for $g=2$, even though $S$ is automatically hyperelliptic, the statement remains true. If $S$ is hyperelliptic and $g>2$ the preceding statement is false and must be replaced by the following: There is a $(2 g-1)$-dimensional subspace $A^{\prime}(S) \subset A(S)$ which is element-wise invariant under $J$ (with suitable notion of invariance), and there exists an index set $I^{\prime}(S)$, analogous to $I(S)$, containing $2 g-1$ index pairs such that $A^{\prime}(S)$ is generated by $d \zeta_{i} d \zeta_{j},(i, j) \in I^{\prime}(S)$. The complement of $A^{\prime}(S)$ in $A(S)$ is a $(g-2)$-dimensional subspace, each element of which changes sign under $J$ and no element of which is generated by products of $d \zeta$ 's.

The following theorem is historically older in its essence than Theorem 2 and contains the first method for providing $T^{\circ}(S)$ with a complex analytic structure.

Theorem 3. Given $S, g>0$, and a canonical homology basis $(\gamma, \delta)$ on it, the map ${ }^{*} \pi: \mathfrak{J}^{\circ}(S) \rightarrow \Im_{。}$ (Proposition 5) is complex analytic. The map ${ }^{*} \pi: T^{\circ}(S) \rightarrow \mathfrak{S}_{0}$ defined as the natural projection $T^{o}(S) \rightarrow J^{\circ}(S)$ (Definition 1) followed by ${ }^{\star} \pi$ is also complex analytic. For $g \geqq 2$, in order to compute the differentials $d^{*} \pi$ at $\left\langle S, S^{\prime}, \alpha\right\rangle \in T^{g}(S)$ and $d^{*} \pi$ at $\left\{S, S^{\prime}, \alpha\right\} \in J^{o}(S)$ consider $\pi\left[S^{\prime}, \alpha(\gamma, \delta)\right]$ (equation (1b), Lemma 3, Proposition 5) and consider a set of coordinates per Prescription I on $S^{\prime}$; then both differentials are given explicitly by the one formula

$$
\begin{aligned}
& d \pi_{i j}\left[S^{\prime}, \alpha(\gamma, \delta)\right] \\
& \quad=\sum_{\alpha=1}^{3 g-3}-(-1)^{1 / 2}\left(d \zeta_{i} / d z d \zeta_{j} / d z, \mu_{\alpha}\right) d c_{\alpha}, i, j=1, \cdots, g,
\end{aligned}
$$

where $z$ is a typical local variable on $S^{\prime}$ and the parenthesis symbol is that of Proposition 3 on $S^{\prime}$. On $T^{o}(S)-U^{o}(S)$ and $\mathrm{J}^{o}(S)-\mathcal{U}^{o}(S),{ }^{*} \pi$ and ${ }^{*} \pi$ have (maximum) rank $3 g-3$ while on $U^{\circ}(S)$ and $\mathcal{U}^{\circ}(S)$ they have rank $2 g-1$. The case $g=2$ is subsumed in the last statement. The case $g=1$ requires only one term on each side of (2) and the rank is everywhere one. The following prescription yields complex analytic structures on $T^{\circ}(S)$ and $J^{\circ}(S)$ equivalent to those of Theorem 2.

PRESCRIPTION II. Let $I\left(S^{\prime}\right)$ be an index set as described in Lemma 5 for $S^{\prime}$, assuming $S^{\prime}$ not hyperelliptic, i.e., $\left\langle S, S^{\prime}, \alpha\right\rangle \in T^{o}(S)-U^{o}(S)$, 
$\left\{S, S^{\prime}, \alpha\right\} \in \mathfrak{J}^{o}(S)-\mathcal{U}^{g}(S)$. Then in a sufficiently small neighborhood ${ }^{*} N$ in the Teichmueller topology of $\left\langle S, S^{\prime}, \alpha\right\rangle$ and its projection $\sharp_{N}$ in $J^{o}(S)$, a neighborhood of $\left\{S, S^{\prime}, \alpha\right\}$, the functions $\pi_{i j}\left[S^{\prime \prime}, \beta(\gamma, \delta)\right],(i, j) \in I\left(S^{\prime}\right),\left\langle S, S^{\prime \prime}, \beta\right\rangle$ a typical element of ${ }^{*} N$, are local coordinates in a complex analytic manifold structure on $T^{\circ}(S)$ $-U^{o}(S)$ and a naturally projected such structure on $J^{o}(S)-\mathcal{U}^{o}(S)$. If $\left.\left\langle S, S^{\prime}, \alpha\right\rangle \in U^{o}(S),\left\{S, S^{\prime}, \alpha\right\} \in \mathcal{U}^{o}(S), g\right\rangle 2$, the preceding statement is false (because of the second part of Lemma 5) and must be replaced by the following one:

Prescription II'. Given any $\left\langle S, S^{\prime}, \alpha\right\rangle \in T^{\circ}(S)$ and the corresponding $\left\{S, S^{\prime}, \alpha\right\} \in \mathcal{J}^{\circ}(S), g \geqq 2$ ( $S^{\prime}$ need not be hyperelliptic), and a sufficiently small neighborhood ${ }^{*} N$ of $\left\langle S, S^{\prime}, \alpha\right\rangle$ and its projection $+N$ in $\Im^{o}(S)$, there exists on $S^{\prime \prime}$, where $\left\langle S, S^{\prime \prime}, \beta\right\rangle$ ranges over ${ }^{*} N$, an abelian differential $d \zeta$ of first kind and complex constants $d_{1}, \cdots, d_{o}$ such that $d \zeta$ has $2 g-2$ distinct zeros $z_{0}, z_{1}, \cdots, z_{2 g-3}$ and

$$
d_{i}=\int_{\beta\left(\gamma_{i}\right)} d \zeta, \quad i=1, \cdots, g,
$$

where $\beta\left(\gamma_{i}\right)$ is the transform of $\gamma_{i}$ on $S$ by $\beta$ and the dependence of $d \zeta$ and $z_{0}, \cdots, z_{2 g-3}$ on $\left\langle S, S^{\prime \prime}, \beta\right\rangle$ and $(\gamma, \delta)$ is suppressed in the interest of simple notation. Then, choosing ${ }^{*} N$ (and ${ }^{*} N$ ) smaller if necessary, one finds that

$$
\begin{aligned}
& \tau_{i}\left[S^{\prime \prime}, \beta(\gamma, \delta)\right]=\int_{\beta\left(\delta_{i}\right)} d \zeta, \quad i=1, \cdots, g \text {, and } \\
& \tau_{\sigma+i}\left[S^{\prime \prime}, \beta(\gamma, \delta)\right]=\int_{z_{0}}^{z_{i}} d \zeta, \quad i=1, \cdots, 2 g-3,
\end{aligned}
$$

are local coordinates in a complex analytic manifold structure on $T^{o}(S)$, and these structures coincide with those of Prescription II on $T^{o}(S)-U^{o}(S)$ and $\jmath^{o}(S)-\mathcal{U}^{o}(S)$. Corresponding to formula (2) and with the same conventions one has, at $\left\langle S, S^{\prime}, \alpha\right\rangle$ and $\left\{S, S^{\prime}, \alpha\right\}$,

$$
\begin{aligned}
d \tau_{i} & =\sum_{\alpha=1}^{30-3}-(-1)^{1 / 2}\left(d \zeta_{i} / d z d \zeta / d z, \mu_{\alpha}\right) d c_{\alpha}, \quad i=1, \cdots, g \\
d \tau_{o+i} & =\sum_{\alpha=1}^{80-3}-(-1)^{1 / 2}\left(d \eta_{i} / d z d \zeta / d z, \mu_{\alpha}\right) d c_{\alpha}, \quad i=1, \cdots, 2 g-3,
\end{aligned}
$$

where $d \eta_{i}$ is the normal differential of third kind on $S^{\prime}$ (i.e., zero periods over $\left.\alpha\left(\gamma_{j}\right), j=1, \cdots, g\right)$ with residue -1 at $z_{0}$ and 1 at $z_{i}$. In addition, the quadratic differentials $d \zeta_{1} d \zeta, \cdots, d \zeta_{0} d \zeta, d \eta_{1} d \zeta, \cdots$, $d \eta_{2 g-3} d \zeta \operatorname{span} A\left(S^{\prime}\right)$. 
The following theorem is of independent interest although not needed in subsequent developments.

THEOREM 3 (BIS). $U^{g}(S), g>2$, is the union of a countable set of relatively closed, isolated complex analytic submanifolds of $T^{\circ}(S)$ of complex dimension 2g-1. An analogous statement holds for $\mathfrak{u}^{g}(S)$ and $\mathfrak{J}^{g}(S)$. $A$ prescription for local coordinates completely analogous to Prescription II holds for $U^{g}(S)$ and น $^{g}(S)$ with the substitution of the index sets $I^{\prime}$ of Lemma 5 for the sets $I$.

C. Conformal automorphisms and the structure of $M^{g}$. To avoid excessive length and complication of statements I shall deal first with the general situation and return later to some special observations about $g=0,1,2$.

Lemma 6. For $g \geqq 2, \alpha: S \rightarrow S, \alpha$ conformal, and $\alpha \approx 1$ (in particular, $\alpha \sim 1) \Rightarrow \alpha=1$. $H(S)$, the group of all conformal automorphisms (selfmaps) of $S$ (composition of maps on the left as usual), is a finite group.

Lemмa 7. Given $f \in H(S)$ and a differential on $S$, for example, $\phi \in A(S)$, define $f \phi \equiv \phi(w(z))(d w(z))^{2}$, where $z$ and w are local parameters at $P \in S$, say, and $f^{-1}(P)$, and $w(z)$ is the local realization of $f^{-1}$. Given a subgroup $G(S) \subset H(S)$, let $R(G, S)$ be the linear representation of $G(S)$ on $A(S)$ defined by $\phi \rightarrow f \phi$. For $g \geqq 2, R(G, S)$ is faithful unless $g=2$ and $S$ is hyperelliptic in which case the kernel is $\{I, J\}$. In addition, if $S$ is not hyperelliptic, the invariant subspace of $R(G, S)$ is of dimension at most $3 g-5$.

Proposition 6. Define ${ }^{* *}: H\left(S^{\prime}\right) \rightarrow \Gamma^{g}(S)$, respectively, \#: $H\left(S^{\prime}\right)$ $\rightarrow \mathfrak{M}^{\circ}(S)$, by ${ }^{* *} f=\left(\alpha^{-1} f \alpha\right)^{*}$ (Proposition 1), respectively $\sharp^{\sharp} f=\left(\alpha^{-1} f \alpha\right)^{\#}$. $A$ necessary and sufficient condition for $\alpha^{*}\left\langle S, S^{\prime}, \beta\right\rangle=\left\langle S, S^{\prime}, \beta\right\rangle$, respectively $\alpha^{\#}\left\{S, S^{\prime}, \beta\right\}=\left\{S, S^{\prime}, \beta\right\}$, where $\alpha^{*} \in \Gamma^{\circ}(S), \alpha^{\#} \in \mathfrak{M}^{\circ}(S)$, $\left\langle S, S^{\prime}, \beta\right\rangle \in T^{o}(S),\left\{S, S^{\prime}, \beta\right\} \in \mathcal{J}^{o}(S)$, is that $\alpha^{*}={ }^{*} f$, respectively $\alpha^{*}=\#^{*} f$, for some $f \in H\left(S^{\prime}\right)$. ** and \#\# are anti-homomorphisms.

At this point I need a consequence of Teichmueller's theorem as noted in $\$ 1$.

Proposition 7. Consider $\left\langle S, S^{\prime}, \alpha\right\rangle \in T^{g}(S)$, respectively $\left\{S, S^{\prime}, \alpha\right\}$ $\in \mathfrak{J}^{\circ}(S)$, for $g \geqq 2$. Given a subgroup $G\left(S^{\prime}\right) \subset H\left(S^{\prime}\right)$, the set $F^{*} \subset T^{g}(S)$, respectively $F^{\sharp} \subset J^{g}(S)$, of points fixed under $* * G\left(S^{\prime}\right)=\Gamma \subset \Gamma^{g}(S)$, respectively \# $G\left(S^{\prime}\right)=\mathfrak{M}$ and sufficiently close to $\left\langle S, S^{\prime}, \alpha\right\rangle$, respectively $\left\{S, S^{\prime}, \alpha\right\}$, is a cell of real dimension equal to twice the complex dimension of the invariant subspace of $R(G, S)$ (Lemma 7).

CoRollary. There exists $S$, for $g>2$, such that $H(S)$ reduces to the 
identity, i.e., $S$ admits no nontrivial conformal automorphism. $\alpha \sim 1 \Leftrightarrow \alpha^{*}$ $=1^{*}$ and $\alpha \approx 1 \Leftrightarrow \alpha^{*}=1^{*}$, where $\alpha: S \rightarrow S$ and $1^{*}$ and $1^{*}$ are identities of $\Gamma^{\circ}(S)$ and $\mathfrak{M}^{\circ}(S)$, respectively. The anti-homomorphisms ** and \#\# are anti-isomorphisms onto the isotropy groups $\Gamma^{o}\left(S, S^{\prime}, \alpha\right) \subset \Gamma^{g}(S)$ and $\mathfrak{M}^{\circ}\left(S, S^{\prime}, \alpha\right) \subset \mathfrak{M}^{g}(S)$ of $\left\langle S, S^{\prime}, \alpha\right\rangle$ and $\left\{S, S^{\prime}, \alpha\right\}$, respectively.

Proposition 8. Given $\left\langle S, S^{\prime}, \alpha\right\rangle \in T^{\circ}(S)$, respectively, $\left\{S, S^{\prime}, \alpha\right\}$ $\in J^{o}(S), g \geqq 2$, there exists a density $\lambda$ on $S^{\prime}$ which is invariant under $H\left(S^{\prime}\right)$ (in the sense of Lemma 7). Using the prescription of Proposition 3 to pass from a basis of $A\left(S^{\prime}\right)$ to one of $B\left(S^{\prime}\right) / N\left(S^{\prime}\right)$, one finds that the latter undergoes the complex conjugate of the representation $R\left(G, S^{\prime}\right)$ for any $G\left(S^{\prime}\right) \subset H\left(S^{\prime}\right)$. Furthermore, if the said basis of $B\left(S^{\prime}\right) / N\left(S^{\prime}\right)$ is used in Prescription II to obtain coordinates near $\left\langle S, S^{\prime}, \alpha\right\rangle$ and $\left\{S, S^{\prime}, \alpha\right\}$, then these coordinates under the action of any subgroup $\Gamma \subset \Gamma^{o}\left(S, S^{\prime}, \alpha\right)$, respectively, $\mathfrak{M} \subset \mathfrak{M}^{g}\left(S, S^{\prime}, \alpha\right)$, undergo the hermitian adjoint of the representation $R\left(G, S^{\prime}\right)$, where ${ }^{* *} G=\Gamma$, respectively, ${ }^{\#} G=\mathfrak{M}$. $R\left(G, S^{\prime}\right)$ is completely reducible; in particular, for a suitable basis of $A\left(S^{\prime}\right)$ and thus a corresponding one of $B\left(S^{\prime}\right) / N\left(S^{\prime}\right)$ the fixed points $F^{*}$ of $\Gamma$, respectively, $F^{*}$ of $\mathfrak{M}$ (Proposition 7 ), near $\left\langle S, S^{\prime}, \alpha\right\rangle$, respectively, $\left\{S, S^{\prime}, \alpha\right\}$, form a complex analytic submanifold on which the coordinates of the noninvariant subspace of the adjoint of $R\left(G, S^{\prime}\right)$ are zero while the coordinates of the invariant subspace are parameters.

TheOREM 4. $M^{o}=\mathfrak{J}^{o}(S) / \mathfrak{M}^{\circ}(S)$ for any fixed $S, g>2$, is a normal analytic space. $\mathrm{J}^{\circ}(S)$ is a branched covering of $M^{\circ}$, where the branch locus $D^{o}(S) \subset \mathfrak{J}^{o}(S)$ is the totality of fixed points of elements of $\mathfrak{M}^{\circ}(S)$ and the description of $D^{\circ}(S)$ near one of its points $\left\{S, S^{\prime}, \alpha\right\}$, as a union of complex analytic submanifolds corresponding to subgroups of $H\left(S^{\prime}\right)$, can be given by the prescriptions of Proposition 8. The points of $M^{\circ}$ on which $D^{\circ}(S)$ projects are all singular (nonuniformizable) points with the following exceptions: all points of $M^{3}$ representing hyperelliptic surfaces without additional automorphisms.

Prescription III. At $\left[S^{\prime}\right] \in M^{0}, g>2$, the local ring of germs of holomorphic functions on $M^{\theta}$ near $\left[S^{\prime}\right]$ is obtained by choosing a set of coordinates per Proposition 8 at $\left\{S, S^{\prime}, \alpha\right\} \in J^{o}(S)$ and choosing those germs of functions there which are invariant under the hermitian adjoint of $R\left(H, S^{\prime}\right)$. In particular, in capsule form, to obtain explicitly a minimal set of generators of $m\left[S^{\prime}\right]$, the maximal ideal of $\Omega\left[S^{\prime}\right]$ containing those elements vanishing at $\left[S^{\prime}\right]$, which set will also be a minimal basis for $m\left[S^{\prime}\right]$, one proceeds as follows. Consider on $S^{\prime}$ a basis for $A\left(S^{\prime}\right)$, the quadratic differentials. Operating on the basis by $R\left(H, S^{\prime}\right)$ (Lemma 7$)$ one obtains a set of $(3 g-3) \times(3 g-3)$ 
matrices $h_{1}, \cdots, h_{n}$, where $n$ is the order of $H\left(S^{\prime}\right)$, representing $H\left(S^{\prime}\right)$ faithfully. Let $h_{1}^{*}, \cdots, h_{n}^{*}\left({ }^{*}\right.$ means hermitian adjoint) act on a set of $3 g-3$ variables $x_{1}, \cdots, x_{3 g-3}$ in the usual way. Let $m$ be the ring of power series in the $x$ 's with complex coefficients and no constant terms, each of which converges in some neighborhood of the origin when the $x$ 's are given complex values and is invariant under all the $h$ 's. There is a finite set of homogeneous nonconstant polynomials in the $x$ 's, $Q_{1}, \cdots, Q_{r}$, each invariant under the $h$ 's such that every element of $m$ is a power series in the $Q$ 's converging in some neighborhood of the origin. Then any irreducible subset of the $Q$ 's (no member a polynomial in the others) has the same number of elements, say $N$, and is a minimal set of generators and a minimal ideal basis of $m$. If one now takes the same basis of $A\left(S^{\prime}\right)$ and forms coordinates $c_{1}, \cdots, c_{30-3}$ near $\left\{S, S^{\prime}, \alpha\right\}$ as per Proposition 8 and Prescription I and sets $x_{1}=c_{1}, \cdots, x_{3 g-3}=c_{3 g-3}$, then $m$ becomes $m\left[S^{\prime}\right]$ and the irreducible set of $Q$ 's the desired basis. If, for the sake of concreteness, one wishes to use Prescriptions II or II', then the bases of $A\left(S^{\prime}\right)$ indicated there are to be used and the $c$ 's replaced by the increments of the corresponding $\pi$ 's (or $\tau^{\prime}$ 's), i.e., $\pi-\pi^{\prime}$, where $\pi$ runs over a neighborhood and $\pi^{\prime}$ is the fixed set of coordinates of $\left\{S, S^{\prime}, \alpha\right\}$.

There are three ingredients in this prescription which have not yet been specified explicitly. (1) Given $H\left(S^{\prime}\right)$ as an abstract group, how does one obtain the matrices $h_{1}, \cdots, h_{n}$ ? (2) How does one compute explicitly the generating polynomials $Q_{1}, \cdots, Q_{r}$ ? (3) Having the $Q$ 's, how does one extract an irreducible set? Let me say that to my knowledge the answer to (3) lies more in the realm of art than science at the present time, that is, there is no known general algorithm; rather it requires a judicious and perhaps tedious choice once questions (1) and (2) have been answered for any given group. However, it is possible to give explicit answers, drawn from function theory, group theory, and algebra, to (1) and (2).

PRESCRIPTION III (ADDENDUM). Given $H\left(S^{\prime}\right)$ as an abstract group, suppose that its irreducible linear representations are known. To determine the representation $R\left(H, S^{\prime}\right)$ on the quadratic differentials of $S^{\prime}$ it is sufficient, given any irreducible representation $\Delta$ of $H\left(S^{\prime}\right)$ of degree $r$, to find its multiplicity $N$. Consider the Riemann surface $S^{-}$of genus $g^{-}$obtained by identifying $S^{\prime}$ under $H\left(S^{\prime}\right) . S^{\prime}$ is an $n$ sheeted covering of $S^{-}$, branched over, say, $P_{1}, \cdots, P_{l} \in S^{-}$. Suppose that, over $P_{i}, S^{\prime}$ has branch points of order $n_{i}$ where $n_{i} \mid n$ (as it will). There is a natural homomorphism of the fundamental group of $S^{-}-\left\{P_{1}, \cdots, P_{l}\right\}$ onto $H\left(S^{\prime}\right)$. The homomorphic image of a simple 
loop about $P_{i}$ is a class of mutually conjugate elements of order $n_{i}$ whose representations in $\Delta$ have $n_{i}$ th roots of unity as eigenvalues. Let $\exp \left(2 \pi i \alpha / n_{i}\right)$ occur $N_{i \alpha}$ times in such a matrix. Then

$$
N=r\left(3 g^{-}-3\right)+\sum_{i=1}^{l} \sum_{\alpha=0}^{n_{i}-1} N_{i \alpha}\left[\left(1-\frac{1}{n_{i}}\right)+\left\langle\frac{1-\alpha}{n_{i}}\right\rangle\right],
$$

where $\langle x\rangle=x-[x]$ is the fractional part of $x$. Knowing the matrices $h_{1}, \cdots, h_{n}$ of $R\left(H, S^{\prime}\right)$, the generating set of invariant homogeneous polynomials can be formed by the following algorithm. If $h_{i}=\left(h_{\alpha \beta}^{i}\right)$, $i=1, \cdots, n, \alpha, \beta=1, \cdots, 3 g-3$ and $x_{1}, \cdots, x_{3 g-3}$ is a set of variables, define

$$
x_{\alpha}^{i} \equiv \sum_{\beta=1}^{30-8} h_{\alpha \beta}^{i} x_{\beta}, \quad i=1, \cdots, n ; \alpha=1, \cdots, 3 g-3,
$$

and define

$$
J_{\mu_{1}} \cdots \mu_{k} \equiv \sum_{i=1}^{n}\left(x_{1}^{i}\right)^{\mu_{1}} \cdots\left(x_{30-3}^{i}\right)^{\mu_{k}} .
$$

Then $J_{\mu_{1} \cdots \mu_{k}}$ for $0<\mu_{1}+\cdots+\mu_{k} \leqq n$ form a generating set of invariant polynomials for $m$ (Prescription III).

The final issue to be dealt with is the concrete embedding of $M^{o}$ which results from the embedding of $T^{o}(S)$ in $\mathbb{S}_{0}$ in Proposition 5.

I recall the definitions of two groups. The homogeneous Siegel modular group, $\operatorname{Sp}(g, Z)$, of degree $g$, is the set of $2 g \times 2 g$ matrices $\mathfrak{M}$, with integral entries, satisfying

$$
\begin{aligned}
& \mathfrak{M}\left(\begin{array}{rr}
0_{o} & I_{\theta} \\
-I_{0} & 0_{\theta}
\end{array}\right) \mathfrak{M}^{T}=\left(\begin{array}{ll}
D & C \\
B & A
\end{array}\right)\left(\begin{array}{rr}
0_{o} & I_{\theta} \\
-I_{o} & 0_{\theta}
\end{array}\right)\left(\begin{array}{ll}
D & C \\
B & A
\end{array}\right)^{T} \\
& =\left(\begin{array}{rr}
0_{g} & I_{g} \\
-I_{0} & 0_{g}
\end{array}\right) \text {, }
\end{aligned}
$$

where $T$ denotes transpose, $0_{g}$ and $I_{\sigma}$ are, respectively, the $g \times g$ zero and unit matrices, and $A, B, C, D$ are $g \times g$ submatrices in the indicated positions. The inhomogeneous Siegel modular group $\mathfrak{M}^{0}$ is isomorphic to $\operatorname{Sp}(g, Z) /\left\{I_{2 g},-I_{2 g}\right\}$, where $I_{2 g}$ is the $2 g \times 2 g$ unit matrix, and acts on $\mathfrak{S}_{0}$ by

$$
\pi^{\prime}=(A \pi+B)(C \pi+D)^{-1} \equiv \mathfrak{M} \circ \pi,
$$

where $\pi, \pi^{\prime} \in \mathfrak{S}_{0}$ and the operations are matrix operations. The homogeneous and inhomogeneous groups both have the multiplicative structures defined by the conventional left composition (matrix multiplication for the homogeneous group). 
Lemma 8. Given a canonical homology basis $(\gamma, \delta)$ on $S, g \geqq 1$, let $\mathfrak{M} \in \operatorname{Sp}(g, Z)$ operate on it on the left as a column vector. Then if $\left(\gamma^{\prime}, \delta^{\prime}\right)$ is any canonical basis on $S$ one has $\left(\gamma^{\prime}, \delta^{\prime}\right)=\mathfrak{M}(\gamma, \delta)$ for some $\mathfrak{M} \in \operatorname{Sp}(g, Z)$ and the resulting map is (up to homology) one-one from $\operatorname{Sp}(g, Z) /\left\{I_{2 g},-I_{2 \theta}\right\}$ to the set of canonical bases. If $\pi=\pi[S,(\gamma, \delta)]$ (equation (1b)) and $\pi^{\prime}=\pi\left[S,\left(\gamma^{\prime}, \delta^{\prime}\right)\right]$, then $\pi^{\prime}$ and $\pi$ are related by $(6)$.

Proposition 9. Given $(\gamma, \delta)$ on $S$, the map $+: \mathfrak{M}^{a}(S) \rightarrow \operatorname{Sp}(g, Z)$ defined by $+\beta^{\sharp}=\mathfrak{M}$, where $\beta: S \rightarrow S$ and $\beta(\gamma, \delta)=\mathfrak{M}(\gamma, \delta)($ Lemma 7$)$, is an anti-isomorphism (the dependence of + on $S$ and $(\gamma, \delta)$ is suppressed). One has ${ }^{*} \pi \beta^{*}=+\beta^{\#} \circ{ }^{*} \pi=\mathfrak{M} \circ{ }^{\#} \pi$ (equation (6)). In particular, ${ }^{\sharp} \pi$ induces a map ${ }^{+} \pi: M^{0} \rightarrow \mathfrak{S}_{g} / \mathfrak{M}^{0}, g>2$ with the following properties: (1) ${ }^{+} \pi$ is one-one into, (2) if $\mathfrak{S}_{g} / \mathfrak{M}^{\circ}$ is provided with its standard structure of analytic space, then ${ }^{+} \pi$ is analytic of rank $3 g-3$ on $M^{\circ}-D^{o}$, where $D^{\circ}$ is the critical locus, i.e., the projection of the branch locus $\mathbb{D}^{\circ}(S)$ (Theorem 4). In addition, + and ${ }^{+} \pi$ are such that (3) at a nonhyperelliptic point $\left\{S, S^{\prime}, \alpha\right\} \in \mathbb{D}^{o}(S),+\mathfrak{M}^{o}\left(S, S^{\prime}, \alpha\right)=\mathfrak{M}^{o}\left({ }^{+} \pi\left[S^{\prime}\right]\right)$, the (full) isotropy subgroup of $\mathfrak{M}^{\circ}$ at ${ }^{+} \pi\left[S^{\prime}\right]$, and the analytic action of $\mathfrak{M}^{\circ}\left(S, S^{\prime}, \alpha\right)$ at $\left\{S, S^{\prime}, \alpha\right\}$ is analytically equivalent to that given by equation (6) where $\mathfrak{M}={ }^{+} \beta^{*}, \beta^{\sharp} \in \mathfrak{M}^{o}(S)$, with the substitution on both sides of $\pi_{k l},(k, l)$ $\notin I\left(S^{\prime}\right)$, as functions of $\pi_{i j},(i, j), \in I\left(S^{\prime}\right)$, per Theorem 3 .

There remains now the task of treating the particular cases $g=0,1$, and 2. The study of these cases is revealing because the relationship of the rather abstract treatment outlined heretofore in the general case to the very concrete traditional treatment available for $g=1$ and to a lesser degree for $g=2$ brings the former down to earth, as it were, and reveals graphically its necessity and the inadequacy of the latter.

TheOREM 5. (i) For $g=0$, given any $S$, one has $T^{0}(S)=J^{0}(S)=M^{0}$ $=\left\{\left[S^{\prime}\right]\right\}$, i.e., a set consisting of one point. (ii) For $g=1$, given any $S$, one has $T^{1}(S)=\mathfrak{J}^{1}(S)=\Im_{1}$ (which is the usual upper half-plane), i.e., the map ${ }^{*} \pi={ }^{*} \pi$ of Proposition 5 is not only one-one but onto. $\Gamma^{1}(S)$ $=\mathfrak{M}^{1}(S)$. Given a canonical homology basis $(\gamma, \delta)$ on $S,+\mathfrak{M}^{1}(S)$ $=\mathrm{Sp}(1, Z)=\Gamma(1)$, the classical homogeneous modular group ( emphasize the anti-isomorphic nature of ${ }^{+}, c f$. Proposition 9). The following modifications of Lemmas 6 and 7, Propositions 6, 7 (and corollary), 8, 9, and Theorem 4 are necessary for $g=1: H(S)$ contains a normal, abelian, transitive, one-complex parameter Lie subgroup $N(S)$ such that $H^{\prime}(S) \equiv H(S) / N(S)$ is finite. The map ${ }^{*}$ (Proposition 6) induces antiisomorphisms of $H^{\prime}\left(S^{\prime}\right)$ onto $\mathfrak{M}^{1}\left(S, S^{\prime}, \alpha\right)$. Furthermore, $H^{\prime}(S)$ contains for any $S$ a canonically determined normal cyclic subgroup of order two, $\{I, J\}$, generated by the sheet interchange (which is not canonically 
determined on $S)$. The image of $\{I, J\}$ in $\mathfrak{M}^{1}(S)$ will be denoted by the same symbol; one has that $\mathfrak{M}^{1}(S) /\{I, J\}$ is anti-isomorphic to $\Gamma(1) /\left\{I_{2},-I_{2}\right\}$, and it is this group that should replace $\mathfrak{M}^{\circ}(S)$ in Theorem 4 for $\mathrm{g}=1$. M $\mathfrak{M}^{1}$ is the classical inhomogeneous modular group and the structure of $M^{1}$ thus resulting from Theorem 4 is identified under ${ }^{+} \pi$ with the classical one of the upper half-plane identified under $\mathfrak{M}^{1}$ with the usual conventions at the vertices of orders 2 and 3 (see the introduction). In particular, there is no singular point of $M^{1}$. (iii) Every $S$ of genus 2 admits a canonical sheet interchange $J$. The image of the normal subgroup $\{I, J\} \subset H(S)$ in $\mathfrak{M}^{2}(S)$ under \#\# will again be denoted by $\{I, J\}$ and again in Theorem $4 \mathfrak{M}^{\circ}(S)$ should be replaced by $\mathfrak{M}^{2}(S) /\{I, J\}$ for $g=2$. The map $\#$ (Proposition 5) of $J^{2}(S)$, any $S$, is one-one onto $\mathfrak{S}_{2}-W$, where $W$ is the set each element of which is equivalent to a diagonal matrix of $\mathfrak{S}_{2}$, under $\mathfrak{M}^{2}$, and ${ }^{+} \pi$ identifies $M^{2}$ with $\left(\mathfrak{S}_{2}-W\right) / \mathfrak{M}^{2}$ with the usual structure. The only singular point of $M^{2}$ is the surface class containing the surface generated by the equation $y^{2}=x^{6}-x$.

Part (ii) can be demonstrated by a careful straightforward asymptotic analysis of the elliptic integral of first kind in the course of which not only the structure of $M^{1}$ but its natural compactification emerges. Although I have not seriously attempted it, it seems possible to do the same for $g=2$. For $g=3$ the dimensions of $J^{3}$ and $\Im_{3}$ still coincide, but the exceptional set has not yet been determined although there are indications that the analogous phenomenon occurs: the exceptional matrices are those equivalent under the inhomogeneous Siegel group to completely reduced matrices. There is a beautiful field of research waiting right here. One might hope, in plain language, up to $g=3$ to dispense with the Teichmueller and Torelli structures altogether and obtain, in addition to $M^{1}, M^{2}$ and $M^{3}$ completely from an asymptotic analysis of the abelian integrals of first kind. But for $g \geqq 4,3 g-3<g(g+1) / 2$, so that on purely dimension-theoretic grounds such a naive procedure is no longer adequate. The best that one has, then, is Theorem 3 and Prescription II. Nonetheless, this still leaves open the question of describing explicitly the set ${ }^{+} \pi M^{o}$ (Proposition 9). Here the hope is, inspired by early work of Schottky, that ${ }^{+} \pi M^{\circ} \subset \Im_{\theta} / \mathfrak{M}^{\theta}$ is locally given by the zeros of a finite set of polynomials in the first order theta-nulls (constants) (vide $\$ 4$ ). Less deep and closer to realization is the hope of explicitly compactifying $M^{\sigma}$ by means of the embedding ${ }^{+} \pi M^{\theta} \subset \mathfrak{S}^{\sigma} / \mathfrak{M}^{\theta}$. Indeed, starting with some remarks of mine, other workers in the field are working out an application of the asymptotic analysis of the periods noted above to the problem.

To conclude, as promised in the introduction, I offer some remarks 
on numerical moduli. By that I mean the naive idea of attaching to each $S$ a set of numbers (obviously at least $3 g-3$ in number for $g \geqq 2$ ) such that (1) the equality of two such sets is a necessary and sufficient condition for the conformal equivalence of the associated surfaces, i.e., one wants global moduli; (2) the numbers are intrinsically defined by the $S$ without reference to any other surface or, to put it another way, one wants absolute moduli not relative moduli; and there should be no arbitrary external normalization of the moduli possible; and (3) the moduli should be complex analytic functions on the structure spaces of this section. The only numerical moduli in the preceding sense for general $g$ known to me are the periods of the normal integrals of first kind, and Proposition 5 and Theorem 3 tell the story-a unique embedding of $J^{o}(S)$ as a point set, independent of $S$, in a fixed unnormalizable number space $\mathfrak{S}_{\jmath}$. These moduli have the particularly interesting feature of being classical function-theoretic objects of great utility and, in particular, given $S$ as the Riemann surface associated with an algebraic equation $f(w, z)=0$, they are computable as complex integrals of explicit algebraic functions of $z$ over explicit broken rectilinear paths in the $z$-plane. On all these counts other local or global moduli that are known, all deriving from uniformization in one way or another are excluded as numerical moduli even though they are usually $3 g-3$ if complex, or $6 g-6$ if real, in number. And there's the rub! The only defect of the periods is that they are $g(g+1) / 2$ in number. Theorem 3 shows how certain subsets of $3 g-3$ of them serve locally as moduli, but it is known that no such set serves globally (see $\$ 3$ for references). It is my conjecture that no proper subset will do, but I have not the slightest idea how to prove it.

3. Comments, references, and hints of proofs. As indicated in $\$ 1$ the essentials of Definitions 1 and 2 and Proposition 1 (with the exception of some points I shall mention shortly) stem from Teichmueller [53]. There are some technical points of an expository character here to which attention should be called in order to establish a liaison with earlier publications. Thus in defining the set $T^{\circ}(S)$ Teichmueller and, following him, Ahlfors (e.g. [1, p. 53] and [5, p. 3]) select a fixed $S$ and define $T^{\circ}(S)$ by means of pairs $\left(S^{\prime}, \alpha\right)$, $\alpha: S \rightarrow S^{\prime}, \alpha$ topological. Bers (e.g. $[10$, p. 351]) demands immediately that $\alpha$ be quasi-conformal. Both authors (cf. Ahlfors, preceding reference) then view any other $T^{\circ}\left(S_{1}\right)$ as a change of frame on $T^{\circ}(S)$ with the frames being related by what is formalized in Definition 2 . Kravetz [25, p. 5] is more consistent; he uses pairs, defines $T^{\circ}(S)$ having fixed $S$ once and for all and considers no admissible maps 
other than self-maps (i.e., in my notation, $\Gamma^{o}(S)$ ) and composes his self-maps of $S$ on the left. All of this is unexceptionable. The trouble (from an expository point of view) arises (in the papers of first three authors) when the time comes to introduce metric and analytic structures on $T^{o}(S)$. An examination of the preceding references (or $[4$, p. 168]) shows that an origin, in fact, in my notation, the origin of Definition 1, is singled out, the Teichmueller distance from it to any other point is defined, and the analytic coordinates in its neighborhood are defined; and then, with a quick shuffle of the feet and a wave of the hand, it is asserted that the structure can be carried to the rest of the space by a change of frame. All this can be made quite precise in the notation of $\$ 2$ : one defines, as in Theorem 1, the distance from $\langle S, S, 1\rangle$ to $\left\langle S, S^{\prime}, \alpha\right\rangle$ for all $T^{\circ}(S)$, i.e., all $S$ simultaneously and then defines the distance between $\left\langle S, S^{\prime \prime}, \beta\right\rangle$ and $\left\langle S, S^{\prime \prime \prime}, \gamma\right\rangle$, say, on $T^{\circ}(S)$ to be the distance between $\beta^{*-1}\left\langle S, S^{\prime \prime}, \beta\right\rangle=\left\langle S^{\prime \prime}, S^{\prime \prime}, 1\right\rangle$ and $\beta^{*-1}\left\langle S, S^{\prime \prime \prime}, \alpha\right\rangle=\left\langle S^{\prime \prime}, S^{\prime \prime \prime}, \gamma \beta^{-1}\right\rangle$ on $T^{\prime}\left(S^{\prime \prime}\right)$. In the process one is forced to use the confusion of topological and differentiable functions of the first member of a triple which occurs in the writings mentioned above by considering 1 in $\left\langle S, S^{\prime \prime}, 1\right\rangle$ to be quasi-conformal. The isometry of admissible maps and, in particular, the elements of $\Gamma^{\circ}(S)$, is automatic. One can proceed similarly in Theorem 2 to introduce coordinates, and again the analyticity of admissible maps is automatic. Upon reconsidering the whole thing, however, one sees that the definitions of Theorems 1 and 2 are less cumbersome and more natural and have the advantage of keeping the first member of a triple in a purely topological role, the second member in a differentiable role, and the third member always a topological map. There is then no need whatsoever to distinguish $\langle S, S, 1\rangle$, say, as an origin and, indeed, one will find no further mention of it in $\$ 2$, and one can even then work with pairs and suppress the $(S)$, having picked $S$ once and for all. However, precisely for the purpose of keeping all functional dependences as explicit as possible and making the preceding remarks I have retained the notation used.

The intermediate position of the Torelli space and the role of the group $\Delta^{\circ}(S)$ in Definition 2 and Proposition 1 were pointed out to me in very valuable conversations of many years ago by Murray Gerstenhaber. The fixed-point-free nature of $\Delta^{o}(S)$ and the consequence (Theorem 2) that $\jmath^{o}(S)$ is a manifold were first observed by me [43, p. 548 , footnote 4 ], [44, p. 23], and I give the simple proof here because the manipulations with the symbols of Definitions 1 and 2 used in it serve as models for those needed to prove several other statements, namely, those of Proposition 1, Proposition 6, and the corol- 
lary (it will be immediately apparent why one needs the first statement of the corollary when one tries to prove the following ones). Namely, suppose $\beta^{*}\left\langle S, S^{\prime}, \alpha\right\rangle=\left\langle S, S^{\prime}, \alpha\right\rangle, \beta: S \rightarrow S$; then by Definitions 1 and 2 that means $\left\langle S, S^{\prime}, \alpha \beta^{-1}\right\rangle=\left\langle S, S^{\prime}, \alpha\right\rangle \Leftrightarrow$ there exists an $f: S^{\prime} \rightarrow S^{\prime}$, conformal, such that $f \sim \alpha\left(\alpha \beta^{-1}\right)^{-1}=\alpha \beta \alpha^{-1}$. But since $\beta^{*} \in \Delta^{o}(S)$, $\beta \approx 1, \alpha \beta \alpha^{-1} \approx 1$ and $\sim \approx$, one has $f \approx 1$. But a lemma due to Hurwitz (cf. Lewittes [29, p. 737, Corollary 1]) asserts that $f \approx 1 \Rightarrow f$ $=1$. Hence $\beta \sim 1$ and $\beta^{*}$ is the identity. The reader can now immediately verify the other statements mentioned.

That brings up subheading B and, first of all, Theorems 1 and 2 and their ancillary Definitions 3-5, Lemmas 1 and 2, and Proposition 3. As indicated in $\$ 1$, Theorem 1 in stronger form is due to Teichmueller, and, indeed, Proposition 2 is the stronger form. Again, I remind the reader that as far as the central fact of Theorem 2 , the existence of a complex analytic manifold structure on $T^{a}(S)$ is concerned, it was first found via Theorem 3 (see \$1). However, as formulated, Theorem 2 is due to Bers in [10]. Moreover, while many of the ideas and even the notation of this section through Theorem 2 occur explicitly in Teichmueller's work, the entire spirit and manner of exposition here are due to Bers, who emphasized the role of the Beltrami equation and its solution theory and devised the important idea of using a basis of Beltrami differentials in proving Theorem 2. A few words will lay bare the essential ideas and motivation. Consider first Definition 4. Suppose one has a $C^{1}$ map with nonvanishing Jacobian $\alpha: S \rightarrow S^{\prime}$. Writing $d w=w_{z} d z+w_{\bar{z}} d \bar{z}=w_{z}\left(d z+\left(w_{\bar{z}} / w_{z}\right) d \bar{z}\right)$ and defining $\mu \equiv w_{z} / w_{z}$, one sees that the Jacobian condition, $\left|w_{z}\right|^{2}-\left|w_{z}\right|^{2}>0$ ( $\alpha$ is assumed to be orientation preserving), says precisely $\left|w_{z}\right|^{2}\left(1-|\mu|^{2}\right)$ $>0$, so that $\alpha$ is quasi-conformal according to Definition 4 where $\mu$ as defined is proper and continuous. To give geometrical meaning to this definition of quasi-conformality fix a point $P$ on $S$ and consider $d z$ as a vector there. Writing $d w=w_{z} d z(1+\mu d \bar{z} / d z)$, and $d z=e^{i \phi}|d z|$, one sees that $|d w|=\left|w_{z}\right|\left|1+\mu e^{-2 i \phi}\right|$ has the maximum $\left|w_{z}\right|(1+|\mu|)$ when $\phi=\frac{1}{2} \arg \mu$ and the minimum $\left|w_{z}\right|(1-|\mu|)$ when $\phi=\frac{1}{2} \arg \mu$ $+\pi / 2$, i.e., at right angles. In loose geometrical terms an infinitesimal circle on $S$ about $P$ goes into an infinitesimal ellipse for which the ratio of major to minor axes is $(1+|\mu|) /(1-|\mu|), \mu$ evaluated at $P$. Calling this expression the dilation at $P, K(\alpha, P)$, one sees that $K(\alpha)$ $=\sup K(\alpha, P)$, sup taken over all $P \in S$. From the geometrical interpretation one sees immediately (considering affine maps on the tangent planes) that the inverse map has the same dilation at every point, hence the same dilation, and the dilation at a point under composition of quasi-conformal maps is multiplicative so that $K(\alpha \beta)$ 
$\leqq K(\alpha) K(\beta)$. These two remarks imply immediately that the Teichmueller distance in Theorem 1 is symmetric and satisfies the triangle inequality once one has dealt with the question of the more general (not $C^{1}$ ) quasi-conformal mappings.

According to Definition 4 the non- $C^{1}$ quasi-conformal maps satisfy the Beltrami equation with generalized derivatives. For this notion see $[11$, p. $90, \S 2]$. That one can operate with the generalized derivatives of the composition of two quasi-conformal maps as with $C^{1}$ maps is contained in $[6$, p. 393, Lemma 10]. The two geometric facts remarked above then follow by the usual computations, i.e., multiplication of $2 \times 2$ matrices of partial derivatives representing affine transformations on tangent spaces.

A proof of Lemma 1 within the class of $C^{1}$ maps, i.e., continuous $\mu$, is laborious but well accessible. However, even with continuous $\mu$ the solution theory of the Beltrami equation is as sophisticated as with discontinuous $\mu$. One could then appeal to a smoother map, but since one is going to deal with general $\mu$ anyway, it is much easier as Bers remarks in [12, p. 216, Proposition 2] to prove Lemma 1 with maps with breaks in the derivatives. Lemma 2 is a consequence of the solution theory in [6] under the hypotheses of Definitions 3 and 4.

The positivity of the Teichmueller metric $(S)$ follows in particular from a simple lemma in the solution theory $[11$, p. 95, F], which shows that from the existence of a sequence of maps $\gamma_{n}: S^{\prime} \rightarrow S^{\prime \prime}$, $n=1, \cdots$, such that $K\left(\gamma_{n}\right) \rightarrow 1$, i.e., such that $\left\|\mu_{n}\right\| \rightarrow 0$, where $\mu_{n}$ is derived from $\gamma_{n}$ per Definition 4, one can conclude that there is a limit map $\gamma: S^{\prime} \rightarrow S^{\prime \prime}$ satisfying in suitable local coordinates the Beltrami equation with $\mu \equiv 0$, i.e., the Cauchy-Riemann equations. That is, $D_{S}\left(\left\langle S, S^{\prime}, \alpha\right\rangle,\left\langle S, S^{\prime \prime}, \beta\right\rangle\right)=0 \Leftrightarrow$ there exists a $\gamma: S^{\prime} \rightarrow S^{\prime \prime}, \gamma$ conformal, $\gamma \sim \alpha \beta^{-1}$, i.e., $\left\langle S, S^{\prime}, \alpha\right\rangle=\left\langle S, S^{\prime \prime}, \beta\right\rangle$. I shall remark here that the Beltrami equation is, in unified complex notation, nothing else but Beltrami's generalized Cauchy-Riemann equations for the Riemannian 2-metric $\lambda|d z+\mu d \bar{z}|^{2}$, where $\lambda$ is a density. The integrability condition $w_{\bar{z} z}=\left(\mu w_{z}\right)_{z}$ is, up to a constant, the Beltrami-Laplace equation for that metric. To check this with a standard source in conventional notation (Blaschke, Chapter 6), write $E d x^{2}+2 F d x d y+G d y^{2}$ $=\frac{1}{2}(E+G)|d z|^{2}+\operatorname{Re}\left\{\left(\frac{1}{2}(E-G)+i F\right) d \bar{z}^{2}\right\}=\lambda|d z+\mu d \bar{z}|^{2}$. Comparing, one obtains two equations for $\lambda, \mu$ from which one derives a quadratic for $\lambda$. Solution leads to $\lambda=(E+G) / 4+\frac{1}{2}\left(E G-F^{2}\right)^{1 / 2}, \mu$ $=\left\{\frac{1}{2}(E-G)+i F\right\} / 2 \lambda$. Insertion in the Beltrami equation and the taking of real and imaginary parts complete the story.

To complete the discussion of Theorem 1, I observe that the isometric nature of the admissible maps is a trivial exercise of the sort 
mentioned earlier. The proper discontinuity is proved (modulo notation) in [25, p. 33, Theorem 7.4]. That leaves the task of establishing connectedness. To that end consider $\left\langle S, S^{\prime}, \alpha\right\rangle,\left\langle S, S^{\prime \prime}, \beta\right\rangle$. By Lemma 1 one can find $\gamma: S^{\prime} \rightarrow S^{\prime \prime}, \gamma \sim \beta \alpha^{-1}$ and quasi-conformal with associated proper $\mu \in B\left(S^{\prime}\right)$ (Proposition 3). Observing that $\gamma: S^{\prime \mu} \rightarrow S^{\prime \prime}$ is conformal (Lemma 2 and Definition 5), one sees that $\left\langle S, S^{\prime \mu}, \alpha\right\rangle$ $=\left\langle S, S^{\prime \prime}, \beta\right\rangle$. Hence it is sufficient to prove that the map $\epsilon \rightarrow\left\langle S, S^{\prime \epsilon \mu}, \alpha\right\rangle$ of $0 \leqq \epsilon \leqq 1$ into $T^{o}(S)$ is continuous. The continuity at $\epsilon=0$ is implied by the argument showing positivity of the metric. To extend the argument to nonzero values one needs a technical device (of great importance in the sequel) due to Ahlfors and Bers.

Namely, consider $\mu_{1}, \mu_{2} \in B\left(S^{\prime}\right)$ and proper, and suppose that 1: $S^{\prime} \rightarrow S^{\mu_{1}}=S^{\prime \prime}, 1: S^{\prime} \rightarrow S^{\prime \mu_{2}}=S^{\prime \prime \prime}$ are realized locally by $u(z, \bar{z})$ and $w(z, \bar{z})$, respectively. Then, using the chain rule for partial derivatives, one verifies easily that $S^{\prime \prime \prime}=S^{\prime \prime}$, where

$$
\rho=\left(\mu_{2}-\mu_{1}\right) /\left(1-\mu_{2} \bar{\mu}_{1}\right)\left(u_{z} / \bar{u}_{z}\right),
$$

all functions being evaluated on $S^{\prime \prime}$ via the inverse of $u(z, \bar{z})$.

Setting $\mu_{1}=\epsilon \mu, \mu_{2}=\left(\epsilon+\epsilon^{\prime}\right) \mu, \epsilon^{\prime}$ real and small, one sees immediately that the former argument (at $\epsilon=0$ ) establishes continuity at $\epsilon^{\prime}=0$, i.e., at the remaining $\epsilon$.

Turning to Proposition 3, I remark that the first statement follows from the Riemann-Roch theorem and the fact that any $\phi$ has $4 g-4$ zeros, $g \geqq 2$. The remaining notions are due to Teichmueller in [53], except for the concept of basis which is due to Bers in [10]. All the assertions are obvious once one has the existence of a density. A very useful (in the sequel) way to establish this is to uniformize $S$ by the unit disk and carry back the Poincaré metric there.

The references for Theorem 2 are [10, pp. 353-357], [3, pp. 184186], and [4, pp. 167-168] for Bers' original approach which is that set forth in Prescription I. A later approach due to Bers and Ahlfors is found in [14] and [5, pp. 4-5] and leads to the stronger result of Proposition 4. Some commentary will be useful in understanding Prescription I. The proper character of $\mu$ for small norm of $c$ follows from Schwarz's inequality, and the continuity of the map follows from (F) above as in the connectedness argument. The one-one and hence homeomorphic character of the map requires deeper considerations, namely a systematic use of the unit disk uniformization. Following Bers in [10, pp. 351-353] (cf. also [11, paragraphs 5, 9, 13], [3, pp. 177-179], and [2, pp. 46-47]), one sets up a one-one correspondence between $T^{o}(S)$ and normalized standard sets of generators of uniformizing Fuchsian groups. For the benefit of the reader 
I indicate the liaison between his notation and mine in the quoted passage. Bers starts, in fact, with $T^{o}\left(S^{\prime}\right)$ ( $S^{\prime}$ is his $\left.S_{0}\right)$, i.e., he uses the origin $\left\langle S^{\prime}, S^{\prime}, 1\right\rangle$. However, one can proceed directly with $T^{\circ}(S)$ as follows: uniformize $S^{\prime}$ to obtain a normalized standard set of generators which one assigns to $\left\langle S, S^{\prime}, \alpha\right\rangle$; given $\left\langle S, S^{\prime \prime}, \beta\right\rangle$ write it, as in a similar situation above, as $\left\langle S, S^{\prime \mu}, \alpha\right\rangle$ for some proper $\mu \in B\left(S^{\prime}\right)$; then, as in the quoted passage, obtain another normalized standard set of generators via $\mu$ and assign that to $\left\langle S, S^{\prime \prime}, \beta\right\rangle$, the assignment being unique as remarked there. The argument on the bottom of p. 353 and p. 354 then completes the proof of the one-one assertion when one observes that the hypothesis of Prescription I that $\mu_{1}, \cdots, \mu_{3 g-3}$ are a basis of $B\left(S^{\prime}\right) / N\left(S^{\prime}\right)$ implies, by the main lemma of p. 354, that they are also a complex basis in the sense of p. 354 .

There remains the task of showing that any two sets of coordinates per Prescription I are complex analytically related. This is done by Bers on pp. 356-357 of the quoted reference. I should like to make only two additional comments to enlighten the reader. The first is that along with the mapping of $T^{o}(S)$ onto normalized standard sets of generators goes an embedding in $R^{6 \sigma-6}$, namely, the coordinates of the generating matrices (taking account of the normalization), and this now becomes central. The second is that equation (11), p. 357 and equation (12) on the same page seem (to me) at first sight a little mysterious. Actually, they stem from (F) above, and I would suggest the following clarification. Given vectors $b$ and $c$, write $e=c-b$, $\mu=c_{1} \mu_{1}+\cdots+c_{3 g-3} \mu_{3 g-3}, \quad \nu=b_{1} \mu_{1}+\cdots+b_{3 g-3} \mu_{3 g-3}, \quad$ and $\lambda=\mu-\nu$ $=e_{1} \mu_{1}+\cdots+e_{3 g-3} \mu_{3 g-3}$, where $\mu_{1}, \cdots, \mu_{3 g-3}$ are a basis of $B\left(S^{\prime}\right) / N\left(S^{\prime}\right)$. By formula $(\mathrm{F})$ one has $S^{\prime \mu}=S^{\prime \prime \rho}$, where $S^{\prime \prime}=S^{\prime \nu}$ and $\rho=\{\lambda /(1-\mu \bar{\nu})\}\left(u_{z} / \bar{u}_{z}\right)$. Expanding $\rho$ for $e$ with small $\|e\|$ (norm) one can write $\rho=n+e_{1} m_{1}(e)+\cdots+e_{3 g-3} m_{3 g-3}(e)$, where $m_{i}(e)$ depends holomorphically on $e$ (the presence of $\mu$ without its conjugate in $\rho$ is critical here) and $\left|m_{i}(e)\right|=O(\|e\|)$ as $\|e\| \rightarrow 0$, and where $n=e_{1} n_{1}+\ldots$ $+e_{3 g-3} n_{3 g-3}, n_{i}=\left\{\mu_{i} /\left(1-|\nu|^{2}\right)\right\}\left(u_{z} / \bar{u}_{z}\right)$. Using results of [6], one can replace (12) by $\left|S^{\prime \mu}-S^{\prime \prime n}\right|=\left|S^{\prime \prime \rho}-S^{\prime \prime n}\right|=O\left(\|e\|{ }^{2}\right)$ and proceed with the remainder of Bers' argument.

Now we come to the complex of ideas surrounding Theorem 3. The basic facts about hyperelliptic surfaces are due to Hurwitz (see [22]). The statement in Lemma 3 about the existence of an involutive map $\alpha$ such that $\alpha(\gamma, \delta)=(-\gamma,-\delta)$ follows from the fact that every $S$ admits a map $\beta$ on a hyperelliptic $S^{\prime}$. Setting $\alpha=\beta^{-1} J \beta$, where $J$ is the involution of $S^{\prime}$, suffices. Proposition 5 is Torelli's theorem as formulated by Weil in [56] (see also the references in \$1). The theorem actually proved states that $\pi[S,(\gamma, \delta)]=\pi\left[S^{\prime},\left(\gamma^{\prime}, \delta^{\prime}\right)\right] \Rightarrow$ there 
exists an $f: S \rightarrow S^{\prime}, f$ conformal, such that $f(\gamma, \delta)=\left(\gamma^{\prime}, \delta^{\prime}\right)$ or $\left(-\gamma^{\prime},-\delta^{\prime}\right)$ (the reverse implication is trivial by Lemma 4). That the result is $2-1$ except on $\mathfrak{u}^{o}(S)$ where it is 1-1 results from Lemma 3. Lemma 5 is a theorem due to Max Noether (cf. [43, p. 560, reference 5]) in the nonhyperelliptic case. In the hyperelliptic case it is proved by explicit computation (cf. [42], [44, p. 22 and p. 27], [2, p. 50]).

Theorem 3 through Prescription II is my own work (in [41] and, more carefully in [44]). The moduli used in Prescription $\mathrm{II}^{\prime}$ were first suggested by Riemann in [46, pp. 120-122], then utilized by Schiffer and Spencer in [49, p. 333, Theorem 7.12.1], and finally cast into the form of Prescription II $^{\prime}$ by Ahlfors in [2], although it should be emphasized that the precise form of equation (4) with the $\mu$ 's does not appear there-rather they follow easily from the technique used in [44, pp. 38-39]. As matters presently stand, Theorem 3 is derived from Theorem 2 as indicated in [44] by means of formulas (2) and (4) and the implicit function theorem, the key point being to show thereby that the coordinates of Prescription II (and $\mathrm{II}^{\prime}$ ) are in oneone correspondence with those of Prescription I and, hence, with the points of a neighborhood of $T^{o}(S)$. However, in invoking the implicit function theorem on p. 28 of [44] there is a gap, namely, equation (2) (or (4)) shows merely that the periods are differentiable functions of the $c$ 's at the origin, whereas one needs differentiability in a neighborhood. But that is easily obtained from the derivation of formulas (2) and (4) given in [44, pp. 38-39] by using formula (F) and a technical device analogous to that in [38, pp. 228-231]. After doing this, one sees immediately from the considerations on pp. 28-29 of [44] that the periods are complex analytic functions of the $c$ 's and one can use the complex form of the implicit function theorem instead of the real form used there. That all this is highly nontrivial is seen from the fact that Ahlfors uses the full strength of Teichmueller's homeomorphism result to prove merely that the periods depend continuously on the $c$ 's $([2, \mathrm{p} .56$, last paragraph and p. 50 , last paragraph of §3], all this being implicit). But having gone so far, I point out that, conversely, Prescription I follows from II and II' by using, respectively, the one-one dependence of all the periods on those in II and Torelli's Theorem and Theorem 7.12.1 of [49] for the coordinates of $\mathrm{II}^{\prime}$ to establish the one-one character of all these coordinates on a neighborhood of $T^{o}(S)$. Theorem 3 (bis) except for the "countable, relatively closed, isolated" is due to me in [42]. That the hyperelliptic locus $U^{o}(S)$ is, in fact, topologically a union of disjoint subspaces of $R^{60-6}$ was known to Teichmueller. That the union is countable (in particular, not finite and not connected) is due to Kravetz in 
[25] where one will also find the preceding statement (cf. also [2, pp. 50-53]).

Turning to heading $\mathrm{C}$, I remark that my observations earlier in this section, together with Lewittes' papers, [29] and [27], will readily dispose of anyone's questions about Lemmas 6 and 7, Proposition 6, and the Corollary to Proposition 7. Coming to Propositions 7 and 8, I consider the latter first. The first sentence follows as remarked above from the existence of the carried back (via uniformization) Poincare density $\lambda$. Let $\phi_{1}, \cdots, \phi_{3 g-3}$ form a basis of $A\left(S^{\prime}\right)$. Set $\mu_{1}=\bar{\phi}_{1} / \lambda, \cdots$, $\mu_{30-3}=\bar{\phi}_{3 g-3} / \lambda$. Given $f \in G\left(S^{\prime}\right)$, set $f \phi_{i}=\sum_{j} a_{i j} \phi_{j}, i=1, \cdots, 3 g-3$ (Lemma 7). Then, clearly, $\lambda$ being invariant, one has $f \mu_{i}=\sum_{j} \bar{a}_{i j} \mu_{j}$, which is the second statement. Setting $\mu=c_{1} \mu_{1}+\cdots+c_{3 g-3} \mu_{3 g-3}$ one finds $f \mu=c_{1}^{\prime} \mu_{1}+\cdots+c_{30-3}^{\prime} \mu_{3 g-3}$, where $c_{i}^{\prime}=\sum_{j} \bar{a}_{j i} c_{j}$, which, coupled with the assertion $f^{* *}\left\langle S, S^{\prime \mu}, \alpha\right\rangle=\left\langle S, S^{\prime f \mu}, \alpha\right\rangle$ (Prescription I) is the third statement. To prove the asserted equation, one observes that it is equivalent to $\left\langle S, S^{\prime \mu}, \alpha \alpha^{-1} f \alpha\right\rangle=\left\langle S, S^{\prime \mu}, f \alpha\right\rangle=\left\langle S, S^{\prime f \mu}, \alpha\right\rangle$ (here $f$ is viewed as a topological map of $S^{\prime \mu}$ on itself), i.e., there exists $g: S^{\prime \mu} \rightarrow S^{\prime f \mu}$ conformal, such that $f \sim g$, where $g$ is now interpreted (Definition 5) as a topological map $g: S^{\prime} \rightarrow S^{\prime}$. But one can prove more; in fact, $f$ interpreted as a topological map $f: S^{\prime \mu} \rightarrow S^{\prime \mu}$ is conformal. Indeed, this follows from the Beltrami equation. Let $z$ and $w$ be local parameters on $S^{\prime}$ at $P$ and $f^{-1}(P)$, respectively. Let $1: S^{\prime} \rightarrow S^{\prime \mu}$ be realized at $f^{-1}(P)$ by $u(w, w)$ satisfying $u_{\bar{w}}=\mu(w) u_{w}$. Let $1: S^{\prime}$ $\rightarrow S^{\prime f \mu}$ be realized at $P$ by $v(z, \bar{z})$ satisfying $v_{\bar{z}}=f \mu(z) v_{z}$, where, per Lemma 7, $f \mu(z)=\mu(w)(d w / d \bar{z})(d z / d w)$, where now $w(z)$ is the local realization of $f^{-1}$. Then with the same use of $w$, the change of variable rule applied to the first Beltrami equation leads to $u_{z}=f \mu(z) u_{z}$, i.e., $u$ and $v$ as functions of $z$ satisfy the same Beltrami equation, i.e., symbolically $u\left(f^{-1}\right)=g^{-1}(v)$, say, where $g$ is analytic with nonzero derivative. That proves the third statement. The complete reducibility of $R\left(G, S^{\prime}\right)$ follows immediately from the finiteness of $G\left(S^{\prime}\right)$. The prescription of the last sentence of Proposition 8 by the above then certainly gives $a$ set of fixed points of $\Gamma$. If, however, one assumes Proposition 7, then by dimenson-theoretic considerations it is clear that it gives all.

That leaves us with Proposition 7 and its corollary. To understand the difficulty here one must see that what is needed is to reverse the preceding argument, i.e., from $f^{* *}\left\langle S, S^{\prime \mu}, \alpha\right\rangle=\left\langle S, S^{\prime \prime}, \alpha\right\rangle$ to conclude that $S^{\prime \prime}=S^{\prime f \mu}$, in particular, if $\left\langle S, S^{\prime \mu}, \alpha\right\rangle$ is fixed, that $f \mu=\mu$; that would show that every fixed point of $f^{* *}$ near $\left\langle S, S^{\prime}, \alpha\right\rangle$ is obtained by the prescription of Proposition 8 and thus, in particular, prove Proposition 7. Now if from the equation one could deduce $g: S^{\prime \mu} \rightarrow S^{\prime \prime}$ 
conformal and $=f$ (as a point map) one could simply revise the entire argument of the preceding paragraph and finish. However, by definition one has only $g \sim f$. Hence, one needs a stronger tool, something that will conclude equality from the weaker assumption of homotopy. But that is precisely Teichmueller's theorem with its uniqueness assertion. Indeed if $t: S^{\prime} \rightarrow S^{\prime \mu}, t=1$ as a point map, is Teichmueller's extremal quasi-conformal map, then $t^{-1} g t: S^{\prime} \rightarrow S^{\prime}$ is extremal quasiconformal and $\sim f$. But, by uniqueness, $t^{-1} g t=f$. Examining Teichmueller's theorem, this implies not that $f \mu=\mu$ but rather $f m=m$, where $m$ is a Teichmueller differential [10, p. 355, paragraph 7], $m=k \phi /|\phi|$, where $0<k<1, \phi \in A\left(S^{\prime}\right)$, and $f \phi=\phi$. In other words, if one forms $m=k\left(\bar{c}_{1}^{\prime} \bar{\phi}_{1}+\cdots+\bar{c}_{30-3}^{\prime} \bar{\phi}_{30-3}\right) /\left|c_{1}^{\prime} \phi_{1}+\cdots+c_{30-3}^{\prime} \phi_{3 g-3}\right|$ and, say, $\phi_{1}, \cdots, \phi_{n}$ span the $f$-invariant subspace of $H\left(S^{\prime}\right)$ then the fixed-point locus of $f^{* *}$ is described by $c_{n+1}^{\prime}=\cdots=c_{30-3}^{\prime}=0$. That proves Proposition 7, but it is not the same as Proposition 8; for if $S^{\prime m}=S^{\prime \mu}$ then the $c_{i}^{\prime}$ are not even differentiable, let alone holomorphic, functions of the $c_{i}$.

The Corollary to Proposition 7 follows from the observation that by Lemma 7 and Proposition 7 and the finiteness of any isotropy subgroup of $\Gamma^{o}(S)$ the totality of fixed points of (nontrivial) elements of $\Gamma^{o}(S)$ is locally a countable union of submanifolds of codimension at least 4. Using Hausdorff's definition of dimension (I am indebted to my esteemed colleague, D. J. Newman, for this ingenious observation) one sees that the fixed points cannot exhaust any neighborhood of $T^{o}(S)$, whence the corollary. Another proof patterned after [2, p. 52, 4], remarks that if $\left\langle S, S^{\prime \prime}, \beta\right\rangle$ is fixed under $h \in \Gamma^{o}(S), h \neq 1^{*}$, and close enough to $\left\langle S, S^{\prime}, \alpha\right\rangle$, then $\left\langle S, S^{\prime \prime}, \beta\right\rangle$ is also fixed under $f^{* *}$ for some $f \in H\left(S^{\prime}\right)$. Indeed, if $t: S^{\prime} \rightarrow S^{\prime \prime}, t \sim \beta \alpha^{-1}$, is the Teichmueller map, with dilation $K$, and if $h=h_{0}^{* *}, h_{0} \in H\left(S^{\prime \prime}\right)$, then $t^{-1} h_{0} t: S^{\prime} \rightarrow S^{\prime}$ is a Teichmueller map with dilation at most $K^{2}$ which is not $\sim 1$. If $K$ is sufficiently small, i.e., $\left\langle S, S^{\prime \prime}, \beta\right\rangle$ sufficiently close to $\left\langle S, S^{\prime}, \alpha\right\rangle$, then (by the proper discontinuity of the mapping-class group) $t^{-1} h_{0} t$ must be conformal, which proves the lemma.

Theorem 4 follows immediately from the representation of $M^{\circ}$ as a quotient space and the results in Cartan's paper [15]. In particular, Prescription III results from Proposition 8 and pp. 91-92 of the Cartan paper, together with the remarks on p. 69 of [37]. The statement about the singular character of the critical locus (the projection of the branch locus) comes from [45], where it is observed that, except for the special cases noted, which are also treated in the same note, the statement follows from the last statement of Lemma 7 and the purity of the branch locus. 
The first formula of Prescription III (Addendum) is taken verbatim from the paper [16], and the second from [36].

Equation (5) defining $\operatorname{Sp}(g, Z)$ is merely a transcription of Lemma 8. Indeed, writing down the conditions that $\mathfrak{M}$ transform a canonical basis to another one gives (5). The peculiar arrangement of $A, B$, $C, D$ is due to the desire to write the matrices in (6) in alphabetical order. In fact, writing $\mathfrak{M}(\gamma, \delta)=(D \gamma+C \delta, B \gamma+A \delta)$ and (equation (1b))

$$
\pi_{i j}^{\prime}=\int_{\Sigma_{k} b_{j k} \gamma_{k}+\Sigma_{k} a_{j k} \delta_{k}} d \zeta_{i}^{\prime}=\sum_{k} b_{j k} \int_{\gamma_{k}} d \zeta_{i}^{\prime}+\sum_{k} a_{j k} \int_{\delta_{k}} d \zeta_{i}^{\prime}
$$

and $d \zeta_{i}^{\prime}=\sum_{l} L_{i l} d \zeta_{l}, L$ nonsingular, one has

$$
\pi_{i j}^{\prime}=\sum_{k, l} b_{j k} L_{i l} \delta_{l k}+\sum_{k, l} a_{j k} L_{i l} \pi_{l k},
$$

using equation (1a). In matrix notation one has $\pi^{\prime}=L B^{T}+L \pi A^{T}$. Similarly, one derives $I_{\theta}=L D^{T}+L \pi C^{T}$. Taking transposes and using the symmetry of $\pi^{\prime}$ and $\pi$ one deduces $\pi^{\prime}=(A \pi+B) L^{T}$ and $L^{r}$ $=(C \pi+D)^{-1}$, whence (6) follows.

Turning to Proposition 9, one sees immediately that + is an antiisomorphism into (Definition 2). That + is onto follows from the more comprehensive theorem to Dehn and Nielsen that every automorphism of the fundamental group of $S$ is induced by a self-map of $S$. To prove the second statement, observe that by Definition 2, Lemma 3 , Proposition 5, and Lemma 8, given $\left\{S, S^{\prime}, \alpha\right\}$ one has to consider the basis $\alpha \beta(\gamma, \delta)$ on $S^{\prime}$ and $\pi\left[S^{\prime}, \alpha \beta(\gamma, \delta)\right]$. But $\alpha \beta(\gamma, \delta)=\alpha \mathfrak{M}(\gamma, \delta)$ $=\mathfrak{M} \alpha(\gamma, \delta)$ because of the linearity of the map induced on $H_{1}(S)$ by $\alpha$. But Lemma 8 implies that $\pi\left[S^{\prime}, \mathfrak{M} \alpha(\gamma, \delta)\right]=\mathfrak{M} \circ \pi\left[S^{\prime}, \alpha(\gamma, \delta)\right]$, which proves the statement. The next statements follow immediately from the preceding one ( $\pi$ "commutes" with the quotient operation by $\mathfrak{M}^{o}(S)$ on $\left.\mathfrak{J}^{o}(S)\right)$ and Theorems 3 and $4\left(\mathcal{U}^{o}(S)\right.$ is in $\left.\mathbb{D}^{o}\right)$.

In Theorem 5, (i) follows immediately from the known topological fact that any orientation-preserving map of a 2 -sphere is homotopic to the identity. (ii) follows from the classical theory of elliptic functions (cf. \$1) and the relevant parts of $\$ 2$. The statement in (iii) anent $\Im_{2}-W$ is known from a study of Torelli's theorem (cf. [55, p. 37 , Satz 2]), that about singularities is in [45]. The rest follows from the relevant parts of $\$ 2$.

For Schottky's result see [50].

That no set of periods per Prescription II will serve as global moduli was proved by Bers in [13]. 
4. Applications. In this section I want to illustrate by some examples drawn from my own work and that of my students the application of the ideas of $\$ 2$ to what seems to me from my reading to be two typical problems in this area.

From the time Riemann first introduced the concept of moduli, one finds repeated reference in his work and that of his successors in algebraic function theory and algebraic geometry of curves to the question as to whether a particular property of a Riemann surface (or algebraic curve) holds for "general moduli" or only for "special moduli." As a general approach to such questions I have proposed [43], [44] that to a surface $S^{\prime}$ of genus $g \geqq 2$ to be "tested" one apply the following procedure: assign to the point $P=\left[S^{\prime}\right] \in M^{o}$ a convenient $t=\left\langle S, S^{\prime}, \alpha\right\rangle$ or $\tau=\left\{S, S^{\prime}, \alpha\right\} \in J^{o}(S$ ) (for some $S$ ) over it. To $t$ (or $\tau$ ) and (some or all of) its neighbors one attaches parameters appropriate to the property and lying in a (sufficiently small) domain such that every set of values therein corresponds to a surface with the desired property. One verifies (if possible) that the $\pi$ 's (Proposition 5 and Theorem 3) are analytic functions of these parameters by means of concrete variational formulae and then one computes the rank of the Jacobian at the values corresponding to $t$ (or $\tau$ ). If the number of parameters is $3 g-3$ and the rank is $3 g-3$ then the property is general by definition. If the number is smaller than $3 g-3$ but the rank is maximal the property holds only for special moduli, again by definition, and one has an explicitly parametrized submanifold of exceptional (Teichmueller or Torelli) surfaces. Another approach to the special moduli situation is to set up appropriate local moduli (see $\$ 1$ ) near $t$ and then write down implicit equations (depending analytically on the moduli) for the property in question. These will describe an analytic set in $T^{o}(S)\left(J^{g}(S)\right)$, indeed, a submanifold if the rank is maximal.

Interestingly enough, the same approach is of value in treating the appearance of moduli in certain more modern questions of function theory, namely, extremal problems of conformal mapping, particularly in the work of Schiffer and those inspired by him. Indeed, those with some familiarity with this research will recognize one major source of the techniques to be demonstrated here and in the problems of the preceding paragraph. The first illustration I cite is precisely in this area and is contained in the paper [38], which is essentially Patt's Yeshiva thesis. It has been pointed out that there is a certain fuzziness in the hitherto existing treatment of a central difficulty in extremal problems on multiply connected surfaces. For example, if one wishes to solve an extremal problem for the coefficients of normal- 
ized schlicht mappings of a multiply connected plane domain by the method of interior variation it is immediately clear that, in general, such variations change the conformal type of the domain so that one is no longer in the set of competing mappings. It is, therefore, necessary to exhibit sufficiently general variations which leave the type unchanged. The first satisfactory handling of this problem to my knowledge is in Patt's paper, where he points out that, more generally, to obtain type-preserving concrete variations of any given character of a surface $S^{\prime}$ of genus $g$ (in the example, $S$ is the double of the plane domain) one proceeds exactly as outlined in the preceding paragraph, using, however, more than $3 g-3$ variational parameters, and sets the $\pi$ 's (or $\tau$ 's) (Theorem 3 ) equal to the constant values they have on $\left\langle S, S^{\prime}, \alpha\right\rangle$. The resulting implicit equations describe a submanifold of type-preserving variations. Patt considers a particular kind of variational process, but the method is immediately applicable to the various known techniques.

My second example belongs to the first-mentioned circle of classical problems and deals with the conditions imposed on the moduli by existence of special divisors of degree at most $g$. To the divisor $\mathfrak{a}=P_{1}{ }^{\lambda_{1}} \cdots P_{n}{ }^{\lambda_{n}}$ on $S$ associate the integers $\operatorname{deg} \mathfrak{a} \equiv \sum \lambda_{i}$, $\operatorname{dim} \mathfrak{a}$ $\equiv$ dimension of the space $L(\mathfrak{a})$ of functions $f$ on $S$ for which $\mathfrak{a} \mid(f)$, and ind $a \equiv$ dimension of the space of differentials $\omega$ on $S$ for which $\mathfrak{a} \mid(\omega)$. The Riemann-Roch theorem then reads $\operatorname{dim} \mathfrak{a}^{-1}=\operatorname{deg} \mathfrak{a}-g+1$ tind $\mathfrak{a}$. In particular, if $\mathfrak{a}$ is integral (all $\left.\lambda_{i} \geqq 0\right), L\left(\mathfrak{a}^{-1}\right)$ is, in plain language, the space of meromorphic functions on $S$ having a pole at $P_{i}$ at most of order $\lambda_{i}$ for $i=1, \cdots, n$ and, hence, $\operatorname{dim} a^{-1} \geqq 1$. If, in addition, $\operatorname{deg} a \leqq g$ then the Riemann-Roch theorem shows that in general, i.e., among divisors on $S$ whose degree equals $\operatorname{deg} \mathfrak{a}, \operatorname{dim} \mathfrak{a}^{-1}$ $=1$. If $\operatorname{dim} \mathfrak{a}^{-1} \geqq 2$, i.e., if $L\left(\mathfrak{a}^{-1}\right)$ contains a nonconstant function, then $\mathfrak{a}$ is special (of degree at most $g$ ). However, if $S$ bears a special divisor $\mathfrak{a}$ with deg $\mathfrak{a} \leqq g$ this may be special in the sense of moduli. To give meaning to this and study the phenomemon, at least locally, I have proposed two devices. In the first I take a nonconstant function $f \in L\left(\mathfrak{a}^{-1}\right)$ and use it to represent $S$ as a branched covering of the Riemann sphere. I then use the branch points as parameters by invoking variational formulae for the $\pi$ 's as functions of them. Now, I consider the differentiable product structure with $\jmath^{\circ}(S)$ and $S_{(d)}$ as factors, where $d=\operatorname{deg} \mathfrak{a}$ and $S_{(d)}$ is the $d$-fold symmetric product of the underlying differentiable manifold of $S$. It is easy to show that by means of any one of several concrete variational methods or a suitable choice of $\mu$ 's one can introduce a structure $V_{(d)}^{o}$ of complex manifolds on the product in such a way that $V_{(d)}^{o}$ is an analytic 
fibre space over the base space $J^{\circ}(S)$ with fibre $S_{(d)}^{\prime}$ over $\tau=\left\{S, S^{\prime}, \alpha\right\}$ $\in \mathfrak{J}^{\circ}(S)$ embedded as a complex submanifold. The structure of $V_{(d)}^{o}$ is generated by local complex analytic product structures of suitable neighborhoods. In particular, the point $(a, \tau) \in V_{(d)}^{o}$ has such a product neighborhood $N$. Variation of the branched representation then describes an analytic set $V$ in $N$ whose dimension is the rank of the Jacobian at $\left\langle S, S^{\prime}, \alpha\right\rangle$. If this rank is maximal, $V$ is a submanifold. If the dimension is $3 g-3$, then $(\mathfrak{a}, \tau)$ is "general," otherwise "special."

In [43] this technique was applied in particular to study the distribution of Weierstrass points. In this connection I wish to call attention to some remarkable work by Lewittes on automorphisms of Riemann surfaces and their relation to Weierstrass points with special relevance to those surfaces which arise from normal subgroups of the modular group $\Gamma(1)$. In particular, two small remarks, byproducts of his investigations, deserve comment here. First, another worker in the field has made the statement that a surface $S$ with only general Weierstrass points, i.e., $g\left(g^{2}-1\right)$ distinct Weierstrass points each with gap sequence $(1, \cdots, g-1, g+1)$ lies in a class $[S] \in M^{\circ}$ which is not a singular point. But Lewittes observes that the surface belonging to the level seven group $\Gamma(7)$, discovered by Klein, has genus $g=3,24$ Weierstrass points each with gap sequence $(1,2,4)$, and admits the group $\Gamma(1) / \Gamma(7)$ containing 168 automorphisms. A glance at Theorem 4 of $\$ 2$ shows that this surface is not one of the lower genus exceptions and is, therefore, in a singular class of $M^{\circ}$. The statement is false with a vengeance. On the other hand, in [43] I showed that any surface with general Weierstrass points gives rise to Teichmueller and Torelli surfaces which are general in the sense of moduli! The second observation is that any normal subgroup $G$ of $\Gamma(1)$ with finite index gives rise to a surface $S^{\prime}$ whose representatives in $M^{o}, J^{o}(S)$, and $T^{o}(S)$ are isolated in the sense that the components in $T^{\circ}(S)$ of the fixed locus of that subgroup of $\Gamma^{\circ}(S)$ which is isomorphic to $\Gamma(1) / G$, the automorphism group of $S^{\prime}$, are precisely the points over $\left[S^{\prime}\right] \in M^{0}$. This follows, using Proposition 8 , from the fact noted by Lewittes that there is no quadratic differential in $S^{\prime}$ invariant under the group.

I have devised a second method (details of which will appear in a forthcoming Yeshiva thesis) for handling special divisors. Again one considers an analytic fibre space $W_{(d)}^{o}$ with base $\mathfrak{J}^{0}(S)$ where the fibre over $\tau=\left\{S, S^{\prime}, \alpha\right\}$ is $S_{(d)}^{\prime} \times S_{(d)}^{\prime}$. Observing that the triple $\left(\mathfrak{a}, \mathfrak{a}^{\prime}, \tau\right)$ $=w$, where $a$ is an integral divisor and $a^{\prime}$ is an equivalent divisor with $\operatorname{deg} \mathfrak{a}=\operatorname{deg} \mathfrak{a}^{\prime}$ ( $\mathfrak{a}$ is the polar divisor, $\mathfrak{a}^{\prime}$ the zero divisor of a function on 
$\left.S^{\prime}\right)$, is a point of $W_{(d)}^{o}$, one sees that Abel's theorem gives implicit equations describing an analytic set of surfaces with pairs of equivalent divisors of order $d$ neighboring $w$ (this in contrast with the explicit parametrization of the preceding method). More explicitly, if

$$
\begin{aligned}
\mathfrak{a} & =P_{1}^{\lambda_{1}} \cdots P_{l}^{\lambda_{l}}, \\
\mathfrak{a}^{\prime} & =Q_{1}^{u_{1}} \cdots Q_{k}^{\mu_{k}}, \quad \lambda_{1}+\cdots+\lambda_{l}=\mu_{1}+\cdots+\mu_{k}=d,
\end{aligned}
$$

are equivalent, there exist integers, $m_{i}, n_{i}, i=1, \cdots, g$, such that

$$
\sum_{\alpha=1}^{l} \lambda_{\alpha} \int_{P_{0}}^{P_{\alpha}} d \zeta_{i}-\sum_{\beta=1}^{k} \mu_{\beta} \int_{P_{0}}^{Q_{\beta}} d \zeta_{i}=m_{i}+\sum_{j=1}^{g} n_{j} \pi_{i j}, \quad i=1, \cdots, g,
$$

where $d \zeta_{i}$ and $\pi_{i j}$ are defined in $\S 2$ and $P_{0}$ is not in $\mathfrak{a}$ or $\mathfrak{a}^{\prime}$ but is otherwise arbitrary and fixed. Applying a concrete variational method due to Schiffer and Spencer [49] and the corresponding variational formulae as developed in [38] one can study the locus on $W_{(d)}^{o}$ defined by these equations. The reader should compare the approaches to the special divisor problem I have sketched here with alternative methods due to Röhrl [48] and Meis [33] who used the theory of analytic spaces and coherent sheaves. I believe that my methods are both more elementary and more explicit and are capable of yielding more detailed results.

A related problem of classic origin (Riemann in [2]) is to determine the conditions imposed on the moduli by the vanishing of an even "theta-null." A modern account of the properties of the Riemann theta function is to be found in Lewittes' paper [30], where, in addition to new results, one will find the first correct function-theoretic proofs of some delicate but hitherto obscure assertions of Riemann. From this paper I borrow the following: one defines the Riemann theta function by

$$
\theta(u, \pi)=\sum_{n} \exp (n \cdot \pi n+2 n \cdot u)
$$

where $n$ ranges over all integral $g$-dimensional column vectors, $u$ is a column of $g$ complex variables, $\pi$ is the period matrix of $\$ 2$, and the dot signifies the usual dot product. Consider the half-period vectors $e=\pi i \epsilon^{\prime} / 2+\pi \epsilon / 2$ where $\epsilon, \epsilon^{\prime}$ are integral $g$-vectors. The first $\pi$ is the usual number and the second is the matrix $\pi$ (multiplied by $\epsilon$ in the usual manner to give a vector). This is embarrassing, but it is unequivocal if one examines the context carefully. $e$ is even or odd according as $\epsilon \cdot \epsilon^{\prime} \equiv 0,1(\bmod 2)$. Defining 


$$
\theta\left[\begin{array}{c}
\epsilon \\
\epsilon^{\prime}
\end{array}\right](u, \pi) \equiv \theta(u+e, \pi) \exp \left(\frac{\epsilon \cdot}{2} \frac{\pi \epsilon}{2}+2 \frac{\epsilon \cdot u}{2}+2 \pi i \frac{\epsilon \cdot \frac{\epsilon^{\prime}}{2}}{2}\right),
$$

one finds an even or odd function of $u$ according as $e$ is even or odd. Hence for even $e, \theta\left[{ }_{e^{\prime}}^{e}\right](0, \pi)$ (or, equivalently, $\theta(e, \pi)$ ) is not necessarily zero. But the theory states that, given $P_{0} \in S^{\prime}, \theta(e, \pi)=0$ is equivalent to the existence of a divisor $a=P_{1}^{\lambda_{1}} \cdots P_{l}^{\lambda_{l}}$ and integers $m_{i}$, $n_{i}, i=1, \cdots, g$, with $\lambda_{1}+\cdots+\lambda_{l}=g-1$ such that

$$
\sum_{\alpha=1}^{l} \lambda_{\alpha} \int_{P_{0}}^{P_{\alpha}} d \zeta_{j}+K_{j}\left(P_{0}\right)=-e_{j}+m_{j} \pi i+\sum_{k=1}^{g} n_{k} \pi_{j k}, \quad j=1, \cdots, g,
$$

where $d \zeta_{j}$ now has the period $\pi i$ over $\delta_{j}$ and $\left(K_{1}\left(P_{0}\right), \cdots, K_{g}\left(P_{0}\right)\right)$ is the vector of Riemann constants with base point $P_{0}$, and $e_{j}$ is the $j$ th component of $e$. Applying on the one hand Riemann's theorem on the index of $\mathfrak{a}$ and on the other the concrete variational method used above, one can investigate the locus $\theta\left[{ }_{\epsilon^{\prime}}{ }^{i}\right](0, \pi)=0$ on $W_{(\theta-1)}^{o}$.

To appreciate the subtlety of this attack consider a more direct one. Setting $\theta\left[{ }_{\epsilon^{\prime}}{ }^{6}\right](0, \pi)=\theta_{\epsilon \epsilon^{\prime}}$, from $\theta_{\epsilon^{\prime}}=0$ one concludes $d \theta_{c \epsilon^{\prime}}$ $=\sum_{i, j}\left(\partial \theta_{\epsilon \epsilon^{\prime}} / \partial \pi_{i j}\right) d \pi_{i j}=0$, where one has written $d \pi_{i j},(i, j) \notin I\left(S^{\prime}\right)$, as a linear combination per Theorem 3 of $d \pi_{i j},(i, j) \in I\left(S^{\prime}\right)$. Consider a one-parameter variation of structure at $P \in S^{\prime}$ per $[38$, p. 234, formula 29], where $v$ is the parameter. Then the last equation becomes $\left(d \theta_{c \epsilon^{\prime}} / d v\right)_{v=0}=\sum_{i, j}\left(\partial \theta_{c \epsilon^{\prime}} / \partial \pi_{i j}\right) \zeta_{i}^{\prime}(P) \zeta_{j}^{\prime}(P)=0$, i.e., a linear relation among the quadratic differentials $d \zeta_{i} d \zeta_{j}$ on $S^{\prime}$ (with periods $\pi=\pi\left[S^{\prime},(\gamma, \delta)\right]$ for some $(\gamma, \delta)$ on $\left.S^{\prime}\right)$. But there is more. Consider, with Riemann, $\theta\left[\epsilon_{\epsilon^{\prime}}^{\epsilon}\right](u, \pi)$ with $u_{i}=\int_{P}^{Q} d \zeta_{i}, i=1, \cdots, g$, resulting in $\theta_{\boldsymbol{c t}^{\prime}}(Q)$. Since $e$ is assumed even, the derivatives $\partial \theta\left[{ }_{\epsilon^{\prime}}{ }^{\prime}\right](0, \pi) / \partial u_{j}=0$, $j=1, \cdots, g$. Hence taking the second partial of $\theta_{\epsilon \epsilon^{\prime}}(Q)$ with respect to $z$, the parameter value of $Q$ in a local parameter at $P$, and setting $Q=P(z=0)$ one finds

$$
d^{2} \theta_{\epsilon \epsilon^{\prime}}(P) / d z^{2}=\sum_{i, j}\left(\partial^{2} \theta\left[\begin{array}{c}
\epsilon \\
\epsilon^{\prime}
\end{array}\right](0, \pi) / \partial u_{i} \partial u_{j}\right) \cdot \zeta_{i}^{\prime}(P) \zeta_{j}^{\prime}(P) .
$$

But using the "heat equations" for $\theta$ :

$$
2 \partial \theta\left[\begin{array}{c}
\epsilon \\
\epsilon^{\prime}
\end{array}\right](0, \pi) / \partial \pi_{i j}=\partial^{2} \theta\left[\begin{array}{c}
\epsilon \\
\epsilon^{\prime}
\end{array}\right](u, \pi) / \partial u_{i} \partial u_{j}, \quad i \neq j,
$$

and

$$
\frac{1}{2} \partial^{2} \theta\left[\begin{array}{c}
\epsilon \\
\epsilon^{\prime}
\end{array}\right](u, \pi) / \partial u_{i}^{2}, \quad i=j
$$


one sees by writing $\sum_{i, j}=2 \sum_{i<j}+\sum_{i=j}$ (using symmetry of the $\pi$ 's and the second partials) that the last expression is precisely 4 times the preceding one whence the former is zero. But this is no surprise since by the Riemann "vanishing theorem" [30, p. 48, Theorem 6], $\theta_{\epsilon \epsilon^{\prime}}=0 \Rightarrow \theta_{\epsilon \epsilon^{\prime}}(Q) \equiv 0$. What is surprising is that $\theta_{\epsilon \epsilon^{\prime}}=0$ should manifest itself as an explicit linear combination of $d \zeta_{i} d \zeta_{j}, i, j=1, \cdots, g$, vanishing on $S^{\prime}$ in two different ways, one purely on the "fibre" $S^{\prime}$, and the other as a consequence of the structure of the zero locus of $\theta_{\epsilon \epsilon^{\prime}}$ on $J^{\circ}(S)$. The point is that if all first partials of $\theta_{\epsilon \epsilon^{\prime}}$ vanish at $\pi$, i.e., at $\left\{S, S^{\prime}, \alpha\right\}$, that is, if $\pi$ is a singular point on the locus $\theta_{\epsilon \epsilon^{\prime}}=0$ in $\mathfrak{S}_{0}$ then the preceding is vacuous. But the Riemann vanishing theorem $[30$, p. 54 , Theorem 8 ] shows that then the divisor $a$ above satisfies ind $\mathfrak{a} \geqq 4$. Thus when ind $\mathfrak{a}>2$ (the generic situation is ind $\mathfrak{a}=2$ ) one needs the subtler method of abelian sums sketched above to investigate $\theta_{\epsilon \epsilon^{\prime}}=0$.

Added in proof. In the context of the paragraph following Theorem 5, A. Mayer and D. Mumford in work still under way have made some progress. In particular, Mayer tells me that $g=3$ is settled. In addition, in connection with $\$ 4$ mention must be made of Mayer's paper [58] on the Riemann vanishing theorem, the first correct treatment, and his characterization of the Torelli sublocus of the Siegel half-plane in genus four by the presence of singularities on the zero locus (on the Jacobi variety) of the theta function.

\section{BIBLIOGRAPHY}

1. L. V. Ahlfors, On quasiconformal mappings, J. Analyse Math. 3 (1953), 1-58.

2. - The complex analytic structure of the space of closed Riemann surfaces, Analytic functions, pp. 45-66, Princeton Univ. Press, Princeton, N. J., 1960.

3. - Some remarks on Teichmueller's space of Riemann surfaces, Ann. of Math. (2) 74 (1961), 171-191.

4. - Curvature properties of Teichmueller's space, J. Analyse Math. 9 (1961), $161-176$.

5. — Teichmueller spaces, Proc. Internat. Congr. Math. Stockholm, 1962, pp. 3-9, Institut Mittag-Leffler, Djursholm, 1963.

6. L. V. Ahlfors and L. Bers, Riemann's mapping theorem for variable metrics, Ann. of Math. (2) 72 (1960), 385-404.

7. L. V. Ahlfors and L. Sario, Riemann surfaces, Princeton Univ. Press, Princeton, N. J., 1960.

8. A. Andreotti, On a theorem of Torelli, Amer. J. Math. 80 (1958), 801-828.

9. W. L. Baily, Jr., On the theory of $\theta$-functions, the moduli of Abelian varieties, and the moduli of curves, Ann. of Math. (2) 75 (1962), 342-381.

10. L. Bers, Spaces of Riemann surfaces, Proc. Internat. Congr. Math. 1958, pp. 349-361, Cambridge Univ. Press, New York, 1960.

11. - Quasiconformal mappings and Teichmueller's theorem, Analytic functions, pp. 349-361, Princeton Univ. Press, Princeton, N. J., 1960. 
12. - Uniformization by Beltrami equations, Comm. Pure Appl. Math. 14 (1961), 215-228.

13. - Holomorphic differentials as functions of moduli, Bull. Amer. Math. Soc. 67 (1961), 206-210.

14. - Correction to "Spaces of Riemann surfaces as bounded domains," Bull. Amer. Math. Soc. 67 (1961), 465-466.

15. H. Cartan, Quotient d'un espace analytique par un groupe d'automorphismes, Algebraic geometry and topology, pp. 90-102, Princeton Univ. Press, Princeton, N. J., 1957.

16. C. Chevalley and A. Weil, Über das Verhalten der Integrale 1. Gattung bei Automorphismen des Funktionenkörpers, Abh. Math. Sem. Univ. Hamburg 14 (1934), 358-361.

17. R. Fricke and F. Klein, Vorlesungen iiber die Theorie der Automorphen Funktionen, Teubner, Leipzig, 1926.

18. H. Grauert, Ein Theorem der Analytischen Garbentheorie und die Moduläraume komplexer Strukturen, Inst. Hautes Etudes Sci. Publ. Math. 5 (1960).

19. L. Greenberg, Maximal fuchsian groups, Bull. Amer. Math. Soc. 69 (1963), 569-573.

20. A. Grothendieck, Techniques de construction en geometrie analytique, Séminaire H. Cartan 13 (1960/1961); Fascicules 1 and 2. 1959.

21. E. Hecke, Mathematische Werke, Vandenhoeck and Ruprecht, Gottingen,

22. A. Hurwitz, Über diejenigen algebraischen Gebilde, welche eindeutige Transformationen in sich zulassen, Mathematische Werke, Bd. I, Birkhäuser, Basel, 1932, pp. 241-259; Über algebraische Gebilde mit eindeutigen Transformationen in sich, ibid. pp. $392-430$.

23. K. Kodaira and D. C. Spencer, Existence of complex structure on a differentiable family of deformations of compact complex manifolds, Ann. of Math. (2) 70 (1959), 145166.

24. P. Koebe et al., Jber. Deutsch. Math.-Verein (1912).

25. S. Kravetz, On the geometry of Teichmueller spaces and the structure of their modular groups, Ann. Acad. Sci. Fenn. 278 (1959).

26. M. Kuranishi, On the locally complete families of complex analytic structures, Ann. of Math. (2) 75 (1962), 536-577.

27. J. Lewittes, Invariant quadratic differentials, Bull. Amer. Math. Soc. 68 (1962), $320-322$.

28. - Gaps at Weierstrass points for the modular group, Bull. Amer. Math. Soc. 69 (1963), 578-582.

29. - Automorphisms of compact Riemann surfaces, Amer. J. Math. 84 (1963), 734-752.

30. - Riemann surfaces and the theta function, Acta Math. 111 (1964), 37-61.

.31. A. M. Macbeath, Fuchsian groups, Mimeographed Notes, Aberdeen.

32. H. Martens, Torelli's theorem and a generalization for hyperelliptic surfaces, Comm. Pure Appl. Math. 16 (1963), 97-110.

33. Th. Meis, Die mimimale Blätterzahl der Konkretisierung einer kompakten Riemannschen Fläche, Schr. Math. Inst. Univ. Münster (1960).

34. D. Mumford, $A$ theorem in geometric invariant theory, Bull. Amer. Math. Soc. 67 (1961), 483-487.

35. R. Nevanlinna, Uniformisierung, Springer, Berlin, 1953.

36. E. Noether, Der Endlichkeitsatz der Invarianten endlicher Gruppen, Math. Ann. 77 (1916), 89-92. 
37. D. G. Northcott, Ideal theory, Cambridge Tract 42 , Cambridge Univ. Press, New York, 1953.

38. C. Patt, Variations of Teichmueller and Torelli surfaces, J. Analyse Math. 11 (1963), 221-247.

39. A. Pfluger, Theorie der Riemannschen Flächen, Springer, Berlin, 1893.

40. E. Picard, Traite d'analyse, Vol. 2, Gauthier-Villars, Paris, 1893.

41. H. E. Rauch, On the transcendental moduli of algebraic Riemann surfaces, Proc. Nat. Acad. Sci. U.S.A. 41 (1955), 42-49.

42. - On the moduli of Riemann surfaces, Proc. Nat. Acad. Sci. U.S.A. 41 (1955), 236-238.

43. - Weierstrass points, branch points, and the moduli of Riemann surfaces, Comm. Pure Appl. Math. 12 (1959), 543-560.

44. - Variational methods in the problem of the moduli of Riemann surfaces, pp. 17-40, Contributions to function theory, Tata Institute of Fundamental Research, Bombay, 1960.

45. - Singularities of the modulus spaces, Bull. Amer. Math. Soc. 68 (1962), 390-394.

46. B. Riemann, Theorie der Abel'schen Funktionen, Gesammelte Mathematische Werke und Wissenschaftlicher, 2nd ed., pp. 88-142, Dover, New York, 1953.

47. - Vorlesungen über die allgemeine Theorie der Integrale algebraischer Differentialen, ibid. Supplement I.

48. H. Röhrl, On holomorphic families of fibre bundles over the Riemannian sphere, Mem. Coll. Sci. Univ. Kyoto Ser. A 33 (1961), 435-477.

49. M. Schiffer and D. C. Spencer, Functionals of finite Riemann surfaces, Princeton Univ. Press, Princeton, N. J., 1954.

50. F. Schottky, Über die Moduln der Thetafunktionen, Acta Math. 27 (1903), 235-288.

51. G. Shimura, Modules des variêtés abeliennes polarisies et functions modulaires. I, II, III, Séminaire H. Cartan 10 (1957/1958); Volume 2.

52. G. Springer, Introduction to Riemann surfaces, Addison-Wesley, Reading, Mass., 1957.

53. O. Teichmueller, Extremale quasikonforme Abbildungen und quadratische Differentiale, Abh. Preuss. Akad. Wiss. Math.-Natur. Kl. 22 (1939), 197 pp.

54. - Bestimmung der extremalen quasikonformen Abbildungen bei geschlossenen orientierten Riemannschen Flächen, ibid. 4 (1943), 42 pp.

55. A. Weil, Zum Beweis des Torellischen Satzes, Nachr. Akad. Wiss. Göttingen, Math.-Phys. Kl. II (1957), 33-53.

56. - Modules des surfaces de Riemann, Séminaire Bourbaki 10 (1957/1958).

57. H. Weyl, Die Idee der Riemannschen Fläche, 2nd ed., Teubner, Stuttgart, 1955.

58. A. Mayer, Special divisors and the Jacobian variety, Math. Ann. 153 (1964), 163-167.

Belfer Graduate School of Science, Yeshiva University 